\title{
HS 0837+4717 - a metal-deficient blue compact galaxy with large nitrogen excess ${ }^{\star}$
}

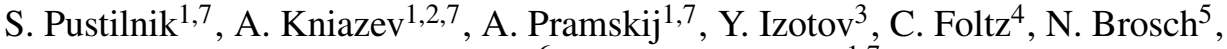 \\ J.-M. Martin ${ }^{6}$, and A. Ugryumov 1,7
}

1 Special Astrophysical Observatory RAS, Nizhnij Arkhyz, Karachai-Circassia 369167, Russia

2 Max Planck Institut für Astronomie, Königstuhl 17, 69117 Heidelberg, Germany

3 Main Astronomical Observatory, 27 Zabolotnoho str., Kyiv 03680, Ukraine

${ }^{4}$ National Science Foundation, 4201 Wilson Blvd., Arlington, Virginia 22230, USA

5 the Wise Observatory and the Raymond and Beverly Sackler Faculty of Exact Sciences, Tel-Aviv University, Tel Aviv 69978, Israel

${ }^{6}$ Observatoire de Paris, place J. Janssen, 92195 Meudon Cedex, France

7 Isaac Newton Institute of Chile, SAO Branch

Received 7 November 2003 / Accepted 18 February 2004

\begin{abstract}
We present the results of high $S / N$ long-slit spectroscopy with the Multiple Mirror and the SAO 6-m telescopes, optical imaging with the Wise 1-m telescope and H I observations with the Nançay Radio Telescope of the very metal-deficient $(12+\log (\mathrm{O} / \mathrm{H})=7.64)$ luminous $\left(M_{\mathrm{B}}=-18 \mathrm{~m} .1\right)$ blue compact galaxy (BCG) HS $0837+4717$. The blue bump near $\lambda 4650$, characteristic of WR stars is detected in the central supergiant H II region, as well as the barely seen red bump at $\lambda 5808$. The derived number of WR stars in the region of the current starburst is $\sim 1000$. Evidence for fast motions in this region is also seen as broad, low-contrast components in the $\mathrm{H} \alpha, \mathrm{H} \beta$ and strong [O III] lines $\lambda \lambda 4959,5007$. While the extinction of the narrow emission lines from the supergiant $\mathrm{H}$ II region is low, the very large Balmer decrement of the broad components suggests that the part of current starburst is highly obscured by dust. Abundance ratios $\mathrm{X} / \mathrm{O}$ for $\mathrm{X}=\mathrm{Ne}, \mathrm{Ar}, \mathrm{S}, \mathrm{Fe}$ and $\mathrm{Cl}$ in the supergiant $\mathrm{H}$ II region are in good agreement with the mean values of other very metal-deficient BCGs. Nitrogen, however, is overabundant by a factor of $\sim 6$. This implies an unusually efficient $\mathrm{N}$ enrichment in HS $0837+4717$, and probably, a non-typical evolution scenario. The $\mathrm{H} \alpha$-line position-velocity $(\mathrm{P}-\mathrm{V})$ diagrams for directions approximately along the major and minor axes reveal disturbed motions of the ionized gas, mainly in peripheral regions. The SW part of the major axis $\mathrm{P}-\mathrm{V}$ diagram looks like a rotation curve, with the velocity amplitude $V_{\text {rot }} \sim 50-70 \mathrm{~km} \mathrm{~s}^{-1}$ at $r=4.3 \mathrm{kpc}$. Its NE part displays, however, strong deviations, indicating either counter-rotation, or a strong outflow/supershell. If it is considered as indicating a shell-like feature its velocity amplitude of $\sim 70 \mathrm{~km} \mathrm{~s}^{-1}$ (relative to the extrapolated rotation curve), and the apparent extent of $\sim 4^{\prime \prime}(3.3 \mathrm{kpc})$ imply a dynamical age of $\sim 14 \mathrm{Myr}$ and the full energetic equivalent of $\sim 2.3 \times 10^{4} \mathrm{SNe}$. The latter indicates continuing starbursts during at least this time interval. The long-slit spectra reveal a complex morphology for this galaxy. It consists of two compact regions at a distance of $\sim 2 \mathrm{kpc}$. Their continuum flux differs by a factor of four. The brightest one is related to the current starburst with the age of $\sim 3.7$ Myr. The slightly redder fainter component could be an older starburst ( 25 Myr). The Wise 1-m telescope $U B V R$ integrated photometry reveals a high optical luminosity for this BCG, and the unusual $(B-V)$ and $(V-$ $R$ ) colours. The morphology of HS $0837+4717$ is highly disturbed, with two small tails emerging to NNW and SSE. Such a disturbed overall morphology, a "double-nucleus" structure, significantly disturbed velocities of ionized gas, together with the very high power of the starburst suggests a possible explanation of the object as a recent merger. We compare the properties of this BCG and of similar objects known in the literature, and conclude that their high nitrogen excess is most probably related to the short phase of a powerful starburst when many WR stars contribute to the enrichment of ISM.
\end{abstract}

Key words. galaxies: starburst - galaxies: abundances - galaxies: interactions - galaxies: evolution - stars: Wolf-Rayet galaxies: individual: HS $0837+4717$

Send offprint requests to: S. Pustilnik, e-mail: sap@sao.ru

* Tables 2-4 are also available in electronic form at the CDS via anonymous ftp tp cdsarc.u-strasbg.fr $(130.79 .128 .5)$ or via http://cdsweb.u-strasbg.fr/cgi-bin/qcat? J/A+A/419/469

\section{Introduction}

Most low-mass gas-rich galaxies have low metallicities, with the typical range of $Z_{\odot} / 15$ to $Z_{\odot} / 3$ (e.g., review of Kunth \& Östlin 2000). Only for a very small fraction of galaxies in the Local Universe is the metallicity extremely low, in the range of $Z_{\odot} / 50$ to $Z_{\odot} / 20$. This range is more characteristic of 
high-redshift damped Ly $\alpha$ absorption systems which could be young galaxies. The study of the extremely metal-deficient (XMD) galaxies allows one to test many theoretical ideas on massive star formation and evolution, and models of galaxy evolution in a very low metallicity environment.

In particular, the abundance patterns of $\alpha$-elements and iron, that is, the ratios $\mathrm{X} / \mathrm{O}$ (with $\mathrm{X}=\mathrm{Ne}, \mathrm{S}, \mathrm{Ar}, \mathrm{Fe}$ ), allow one to check models of stellar nucleosynthesis. For metal-poor galaxies they were found to be remarkably constant over a wide range of $\mathrm{O} / \mathrm{H}$ (e.g., Izotov \& Thuan 1999, hereafter IT99). This implies that all these elements are primary and are produced in the same massive stars. The ratio N/O was also found to be fairly constant for XMD BCGs (IT99), implying mainly primary production of nitrogen in very low metallicity environment.

Wolf-Rayet (WR) stars - a specific very short phase of massive star evolution, characterized by strong mass outflow, and enriched mainly in $\mathrm{N}$ and $\mathrm{C}$, are often seen in high $S / N$ spectra of starburst galaxies (e.g., Kunth \& Joubert 1985; Izotov et al. 1996; Guseva et al. 2000). These stars are detected through the broad emission features of the so called "blue bump" near $\lambda 4650 \AA$, and more rarely in the red, near $\lambda 5808 \AA$. Models of stellar evolution predict that the number of WR stars in a young star cluster is a sensitive function of metallicity, IMF and starburst age (e.g., Schaerer \& Vacca 1998, hereafter SV98).

WR star outflows could produce a significant $N$ overabundance at the locations of young starbursts. However, the analysis of a large sample of H II galaxies by Kobulnicky \& Skillman (1996) showed that galaxies, with and without strong WR features in their integrated spectra, show identical N/O ratios. This is probably explained by nitrogen outflow in the hot phase. Therefore, only significantly after its ejection could the nitrogen be cooled and mixed into the optically observed ionized gas.

Significant nitrogen overabundance is detected in a few starburst galaxies. The most prominent one is Mkn 996 (Thuan et al. 1996), with $12+\log (\mathrm{O} / \mathrm{H})=8.0$. It is a non-typical $B C G$ due to the large number of WR stars and to a powerful outflow from the compact central SF burst. Its N/O in the region with diameter of $0.6 \mathrm{kpc}$ is 4 to 25 times higher than the typical value for low-metallicity BCGs of $\sim 1 / 40$. The morphology of Mkn 996 implies that likely it is a remnant of a recent merger. The authors argue that the observed large N/O ratio is directly related to the powerful outflows of numerous WR stars.

Another well known case is a factor of three nitrogen overabundance, found in two compact regions $(d \sim 20 \mathrm{pc})$ near the central starburst in the nearby $(D \sim 4 \mathrm{Mpc})$ dwarf WR galaxy NGC 5253 (e.g., Kobulnicky et al. 1997, and the summary of previous results therein).

Only three galaxies: UM 420, Mkn 1089, and UM 448, all luminous and with detected WR lines (Guseva et al. 2000), from the sample of 50 BCGs in IT99, show nitrogen overabundance of a factor of $\approx 3$ relative to the mean $\mathrm{N} / \mathrm{O}$ value of $1 / 30$ for their range of $\mathrm{O} / \mathrm{H})$. Finally, the galaxy Haro 11 from the sample of luminous BCGs (Bergvall \& Östlin 2002), also with strong WR bump, shows an N/O ratio that is 6 times higher than for the main BCG group.
In this paper we present the results of a comprehensive study of HS $0837+4717$, found in the Hamburg/SAO survey (Pustilnik et al. 1999). The very low metallicity of HS $0837+4717\left(Z \approx Z_{\odot} / 20\right)$ was first derived from the observations with the SAO RAS 6-m telescope (BTA) in 1996 (Kniazev et al. 2000a). Classified as an XMD BCG, it is unusually luminous for its metallicity. This galaxy shows WR bumps and broad components of strong emission lines of hydrogen and oxygen. This is the next most nitrogen overabundant galaxy known after Mkn 996. But, in contrast to the latter, its optical spectrum does not look atypical for BCGs, and its very large nitrogen overabundance is seen on an otherwise more or less typical BCG background.

In Sect. 2 we describe the observations and their reduction. In Sect. 3 all the results from the reduction and preliminary analysis are presented, including the heavy element abundances, the WR and broad components of strong lines, the ionized gas kinematics and the morphology and photometry. In Sect. 4 we discuss the obtained results and derived parameters of this galaxy, compare its properties with those of other luminous and XMD BCGs, and draw conclusions. The adopted distance to the galaxy is $170.5 \mathrm{Mpc}$; respective scale is 827 pc per arcsecond.

\section{Observations and data reduction}

\subsection{Spectrophotometry and kinematic data}

Spectrophotometric observations of HS $0837+4717$ with high $S / N$ ratio were obtained with the MMT on May 1, 1997. The observations were performed with the Blue Channel of the MMT Spectrograph using a highly-optimized Loral $3072 \times$ 1024 CCD detector. A 1 .. $5 \times 180^{\prime \prime}$ slit was used along with a 500 grooves $\mathrm{mm}^{-1}$ grating used in the first order and with the L-38 second order blocking filter. This yields a spatial scale along the slit of $0^{\prime} 3$ pixel $^{-1}$, a scale perpendicular to the slit of $1.9 \AA$ pixel $^{-1}$, a spectral range $3600-8300 \AA$ and a spectral resolution of $\sim 7 \AA(F W H M)$. For these observations the CCD rows were binned by a factor of 2 , yielding a final spatial sampling of $0{ }^{\prime} 6 \mathrm{pixel}^{-1}$. The observations cover the full spectral range in a single frame which contains all the lines of interest. Furthermore, the spectra have sufficient spectral resolution to separate [O III] $\lambda 4363$ from the nearby $\mathrm{H} \gamma$ and to distinguish between the narrow nebular and the broad WR emission lines. The total exposure time was $40 \mathrm{~min}$, split into 2 equal subexposures, to allow for more effective cosmic ray removal. Both exposures were taken at an airmass of $\sim 1.13$. The slit was oriented in the direction with the position angle $\mathrm{PA}=16^{\circ}$, along the major axis of the brightest region of the galaxy (Fig. 1). The spectrophotometric standard star HZ 44 (Massey et al. 1988) was observed for flux calibration. Reference spectra of He-NeAr were obtained before and after each observation to provide wavelength calibration.

Observations with the BTA were conducted on February 19, 2002 with the Long-slit spectrograph (Afanasiev et al. 1995) at the prime focus of telescope. A CCD detector Photometrics PM1024 with $24 \times 24 \mu \mathrm{m}$ pixel was used for data acquisition. For abundance study a 
Table 1. Journal of spectral observations of HS $0837+4717$.

\begin{tabular}{lccccccc}
\hline \hline Telescope & Date & $\begin{array}{c}\text { Exposure } \\
\text { time }[\mathrm{s}]\end{array}$ & $\begin{array}{c}\text { Wavelength } \\
\text { Range }[\AA]\end{array}$ & $\begin{array}{c}\text { Dispersion } \\
{[\AA / \text { pixel }]}\end{array}$ & $\begin{array}{c}\text { Seeing } \\
{[\operatorname{arcsec}]}\end{array}$ & Airmass & $\begin{array}{c}\text { PA } \\
\text { [degree] }\end{array}$ \\
\hline MMT & $(2)$ & $(3)$ & $(4)$ & $(5)$ & $(6)$ & $(7)$ & $(8)$ \\
BTA & 01.05 .1997 & $2 \times 1200$ & $3600-8300$ & 1.9 & 1.6 & 1.13 & 16 \\
BTA & 19.02 .2002 & $2 \times 1800$ & $3700-6100$ & 2.4 & 2.2 & 1.27 & 44 \\
\hline
\end{tabular}

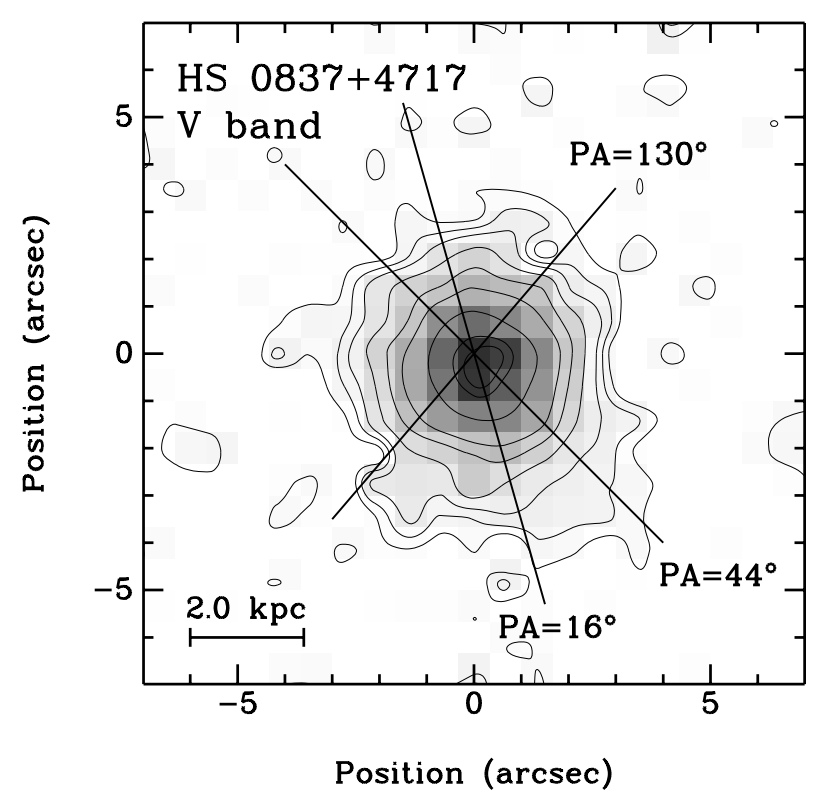

Fig. 1. Morphology of HS $0837+4717$ as seen in the Wise $V$-band image. North is up, East is to the left. The positions of MMT (PA = $\left.16^{\circ}\right)$ and $\mathrm{BTA}\left(\mathrm{PA}=44^{\circ}\right.$ and $\left.130^{\circ}\right)$ long slits are shown. The isophotes sample the image at the brightness levels of 11, 20, 30, 50, 75, 100, 150,200 and 220 units. The minimal level corresponds to $\sim 1.5 \sigma$ of the noise. See Sect. 3.5.

grating with 651 grooves $\mathrm{mm}^{-1}$ was used in the first order, giving $2.38 \AA \operatorname{pixel}^{-1}(F W H M=7 \AA)$ and allowing the simultaneous coverage of the range 3700-6100 $\AA$. Two 30-min exposure spectra were averaged to yield the final spectrum. PA $=44^{\circ}$ was selected to be close to the apparent major axis of the object's fainter isophotes. To complement the study of emission lines in the blue, and to study the ionized gas kinematics, we obtained two spectra in the red with a grating with 1302 grooves $\mathrm{mm}^{-1}$, giving $1.2 \AA \mathrm{pixel}^{-1}$, and $F W H M=3.8 \AA$. These spectra covered the range $6000-7200 \AA$, and were obtained also with the slit direction along PA $=44^{\circ}$, and in the nearly perpendicular direction of $\mathrm{PA}=130^{\circ}$. The long slit of $2^{\prime \prime} \times 120^{\prime \prime}$ was used for both observations.
The reduction of the spectral observations was carried out at SAO using the IRAF $^{1}$ and the MIDAS ${ }^{2}$ software packages. It included bias subtraction, cosmic ray removal, and flat-field correction using exposures of a quartz incandescent lamp. After wavelength mapping, night sky background subtraction, and correcting for atmospheric extinction, each frame was calibrated to absolute fluxes. One-dimensional spectra were extracted by summing up, without weighting, different numbers of rows along the slit depending on exact region of interest. The MMT spectrum of the brightest region of HS $0837+4717$ in the range $3600 \AA<\lambda<7300 \AA$ for the aperture 1 .' $5 \times 3$ '. 0 and the BTA spectra in the blue and red, extracted with the aperture $2^{\prime \prime} \times 2$ ". 8 , are shown in Fig. 2 .

\subsection{Photometry data}

Photometric observation of HS $0837+4717$ were performed with the Wise observatory $1-\mathrm{m}$ telescope on nights 27 and 28.05.1999 in $U, B, V$ and $R_{\mathrm{c}}$ bands. The images were exposed through the standard filters onto a Tektronics $1024 \times$ 1024 pixels CCD and 11'.9 $\times 11$ '.9 field-of-view (at 0.696 arcsec pixel $^{-1}$ ). During the first night, the observations were performed at airmasses of 1.69 till 2.3 , beginning from the $U$-band, and ending with the $R_{\mathrm{c}}$-band. The exposure time was $30 \mathrm{~min}$ for $U$, and $15 \mathrm{~min}$ for the others filters. For the second night, the observations were collected at the airmasses from 1.54 to 2.20 , with the same exposure times. Photometric standards from the fields of Landolt (1992) were observed at an airmass range close to that of the studied galaxy. The seeing during both nights was $\sim(2-2.5)^{\prime \prime}$.

The reduction of photometry was performed in IRAF, following the standard pipe-line, including the de-biasing, cosmic ray removal and flat-fielding. Aperture photometry was performed in circular apertures, and the asymptotic values of the growth curves were accepted as the final integrated magnitudes. The nights were not perfect; some cirrus clouds presumably were present. Therefore, the maximal differences, obtained for the magnitudes in these 2 nights reach 0.3

\footnotetext{
${ }^{1}$ IRAF: the Image Reduction and Analysis Facility is distributed by the National Optical Astronomy Observatory, which is operated by the Association of Universities for Research in Astronomy, In. (AURA) under cooperative agreement with the National Science Foundation (NSF).

${ }^{2}$ MIDAS is an acronym for the European Southern Observatory package - Munich Image Data Analysis System.
} 

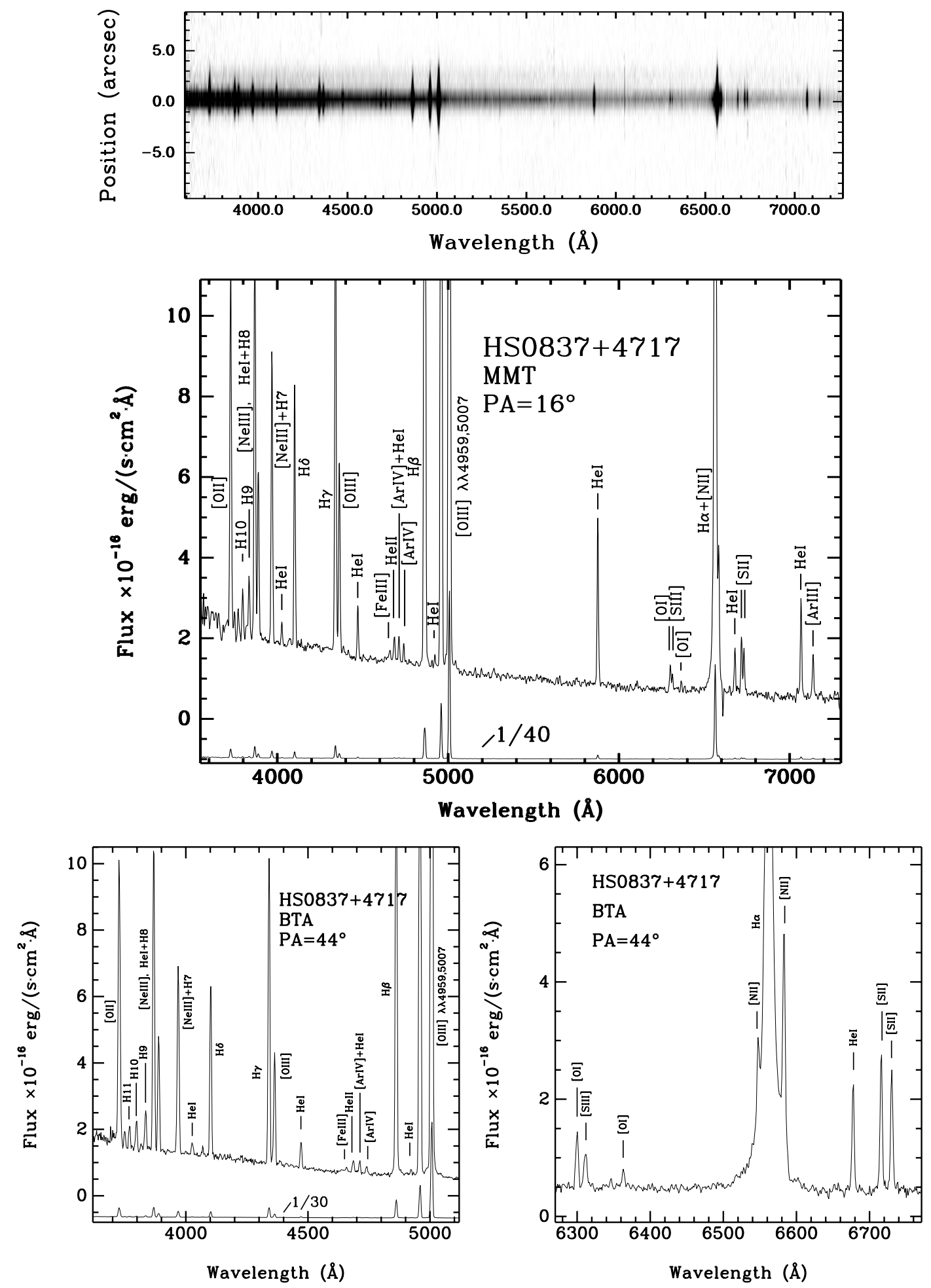

Fig. 2. Upper panel: the MMT 2D spectrum of HS $0837+4717$ with PA $=16^{\circ}$, plotted with the contrast level adjusted so as to show the distribution of the continuum intensity along the slit. SSW is up. While the intensity of strong lines decreases symmetrically relative to the continuum brightness peak, the continuum intensity at the lower level is skewed to the SSW direction, indicating a slightly non-central position of the starburst. Middle panel: de-redshifted MMT 1D spectrum of the bright H II region in HS 0837+4717, extracted with the aperture of 1".5 $\times 3$ '.'0. Bottom panel: de-redshifted 1D blue and red BTA spectra. Note the good consistency between the MMT and BTA spectra.

(in the $V$-band). We combined the results for both nights in the attempt to obtain more reliable magnitudes of this galaxy. We also used as an independent control, the $B V R$ magnitudes and colours derived from the convolution of the MMT spectrum with the $B, V$ and $R$ passbands. 


\subsection{HI data}

H I line observations were carried out in February 1998 with the $300 \mathrm{~m} \mathrm{Nançay}^{3}$ radio telescope (NRT). The NRT has a half-power beam width of $3.7(E W) \times 22^{\prime}(\mathrm{NS})$ at the declination $\operatorname{Dec}=0^{\circ}$.

Since HS $0837+4717$ had a known optical redshift, we split the 1024-channel autocorrelator in two halves and used a dual-polarization receiver to increase the $S / N$ ratio. Each correlator segment covered a $6.4 \mathrm{MHz}$ bandwidth, corresponding to a $1350 \mathrm{~km} \mathrm{~s}^{-1}$ velocity coverage, and was centered at the frequency corresponding to the optical redshift. The channel spacing was $2.6 \mathrm{~km} \mathrm{~s}^{-1}$ before smoothing and the effective resolution after averaging pairs of adjacent channels and Hanning smoothing was $10.6 \mathrm{~km} \mathrm{~s}^{-1}$. The system temperature of the receiver was $\approx 40 \mathrm{~K}$ in the horizontal and vertical linear polarizations. For the target galaxy declination the telescope gain was $0.88 \mathrm{~K} / \mathrm{Jy}$. The observations were made in the standard total power (position switching) mode with 1-min on-source and 1-min off-source integrations. The data were reduced using the NRT standard packages, written by the observatory's staff. The Horizontal and Vertical linear polarization spectra were calibrated and processed independently, and were averaged together.

\section{Results}

\subsection{Chemical abundances}

To derive element abundances of species $\mathrm{O}, \mathrm{Ne}, \mathrm{N}, \mathrm{S}, \mathrm{Ar}$ and $\mathrm{Cl}$ we use the standard method from Aller (1984), and follow the procedure described in detail by Pagel et al. (1992) and Izotov et al. (1994, hereafter ITL94). The electron temperature $T_{\mathrm{e}}$ is known to be different in high- and low-ionization H II regions (Stasińska 1990). In the following we have chosen to determine $T_{\mathrm{e}}(\mathrm{O}$ III $)$ from the $[\mathrm{O}$ III $] \lambda 4363 /(\lambda 4959+\lambda 5007)$ ratio using the five-level atom model of $\mathrm{O}$ III, and $\mathrm{N}_{\mathrm{e}}\left(\mathrm{S}_{\mathrm{II}}\right)$ from the [S II] $\lambda 6717 / \lambda 6731$ ratio. We then adopt $T_{\mathrm{e}}(\mathrm{O}$ III $)$ for derivation of $\mathrm{O}^{2+}, \mathrm{Ne}^{2+}$ and $\mathrm{Ar}^{3+}$. To derive the electron temperature for $\mathrm{O}^{+}$ion, we used the relation between $T_{\mathrm{e}}(\mathrm{O}$ II $)$ and $T_{\mathrm{e}}(\mathrm{O}$ III $)$ fitted by ITL94 for the photoionized H II models of Stasińska (1990). $T_{\mathrm{e}}\left(\mathrm{O}\right.$ II) has been used to derive the $\mathrm{O}^{+}$, $\mathrm{N}^{+}$and $\mathrm{Fe}^{+}$ionic abundances. For $\mathrm{Ar}^{2+}$ and $\mathrm{S}^{2+}$ we have used an electronic temperature intermediate between $T_{\mathrm{e}}(\mathrm{O}$ II $)$ and $T_{\mathrm{e}}(\mathrm{O}$ III) following the prescription of Garnett (1992).

Total element abundances have been derived after correction for unobserved stages of ionization as described by ITL94. For the case applicable to HS $0837+4717$, when the strong nebular He II $\lambda 4686$ emission line is present, this implies that a non-negligible amount of $\mathrm{O}^{3+}$ ion should present. Its abundance is derived from the relation:

$\mathrm{O}^{3+}=\mathrm{He}^{2+} / \mathrm{He}^{+}\left(\mathrm{O}^{+}+\mathrm{O}^{2+}\right)$.

3 The Nançay Radioastronomy Station is part of the Paris Observatory and is operated by the Ministère de l'Éducation Nationale and Institut des Sciences de l'Univers of the Centre National de la Recherche Scientifique.
Then, the total oxygen abundance is equal to

$\mathrm{O}=\mathrm{O}^{+}+\mathrm{O}^{2+}+\mathrm{O}^{3+}$

In Table 2 we present parameters of the $\mathrm{H}$ II region on the emission extracted with the aperture $11^{\prime \prime} 5 \times 33^{\prime \prime} 0$ on the MMT spectrum, and with the aperture $2^{\prime \prime} \times 2$.' 8 - on the BTA spectra. They include both the observed line intensities and intensities corrected for interstellar extinction, relative to that of $\mathrm{H} \beta$ along with the equivalent width $E W(\mathrm{H} \beta)$, observed flux of the $\mathrm{H} \beta$ emission line, and extinction coefficient $C(\mathrm{H} \beta)$. To correct for extinction we used the Whitford (1958) reddening law for our Galaxy.

The derived values of $T_{\mathrm{e}}(\mathrm{O}$ III $), T_{\mathrm{e}}(\mathrm{O}$ II $), T_{\mathrm{e}}(\mathrm{S} \mathrm{III})$ and $N_{\mathrm{e}}(\mathrm{S}$ II $)$ for this supergiant $\mathrm{H}$ II region are shown in Table 3. The ionic and total abundances in the table are derived from the two MMT spectra according to the methods described in ITL94 and in Izotov et al. (1997a, hereafter ITL97). Since they showed a mutual consistency, we averaged their derived parameters.

In the derivation of the $\mathrm{O}, \mathrm{N}$ and $\mathrm{Fe}$ abundances, the broad low intensity underlying components of the strongest emission lines $\mathrm{H} \alpha, \mathrm{H} \beta$ and [O III] $\lambda \lambda 4959,5007$, and WR broad lines were taken into account (see Sect. 3.2). For the abundance determination only the fluxes of the narrow (instrumental width) components of these strong lines were used. In the derivation of the $\mathrm{Cl}$ abundance, we used the respective formula from Aller (1984) with the ICF adopted from Stasińska (1990). The abundances found here are well consistent with the previous results from BTA those in Kniazev et al. (2000a) and those based on the SDSS spectra (Kniazev et al. 2003).

As evident from Table 3, the object belongs to the group of the most metal-poor H II-galaxies, which includes only 1-2 per cent of the known BCGs. Notice the very high abundance of nitrogen. The $\mathrm{N} / \mathrm{O}$ ratio is $\sim 6$ times higher than the typical value for all known XMD BCGs. As shown in IT99, BCGs with $12+\log (\mathrm{O} / \mathrm{H}) \lesssim 7.60$ have $\log (\mathrm{N} / \mathrm{O}) \approx-1.60$ with a very small scatter (Fig. 4). The relative abundances of the other elements in HS $0837+4717$ are close to the mean values for the XMD BCGs (IT99).

\subsection{WR features and broad components of strong emission lines}

The Wolf-Rayet features of the blue bump (near $~ 4650 \AA$ ) and broad low-contrast components of the strong emission lines $\mathrm{H} \beta, \mathrm{H} \alpha$, [O III] $\lambda \lambda 4959,5007$ are evident in both the MMT and BTA spectra of HS $0837+4717$. This allows us to check the accuracy of the derived parameters.

\subsubsection{WR blue bump features}

In Fig. 3 we show an expanded view of the BTA spectrum with the Gaussian decomposition of the narrow lines and the broad component of He II $\lambda 4686$ and the WR lines N III $\lambda 4640$, attributed to late WN stars, and C IV $\lambda 4658$, attributed to WC stars (e.g., SV98). The latter also could appear as the red bump centered at $\lambda 5808$. This bump, however, 
Table 2. Line intensities in the supergiant H II region.

\begin{tabular}{|c|c|c|c|c|}
\hline \multirow[b]{2}{*}{$\lambda_{0}(\AA)$ Ion } & \multicolumn{2}{|c|}{ MMT $\left(1^{\prime \prime} .5 \times 3 .^{\prime \prime} 0\right)$} & \multicolumn{2}{|c|}{$\operatorname{BTA}\left(2^{\prime \prime} \times 2^{\prime \prime} .8\right)$} \\
\hline & $F(\lambda) / F(\mathrm{H} \beta)$ & $I(\lambda) / I(\mathrm{H} \beta)$ & $F(\lambda) / F(\mathrm{H} \beta)$ & $I(\lambda) / I(\mathrm{H} \beta)$ \\
\hline 3727 [O II] & $0.380 \pm 0.019$ & $0.402 \pm 0.022$ & $0.379 \pm 0.021$ & $0.407 \pm 0.024$ \\
\hline 3835 H9 & $0.052 \pm 0.005$ & $0.082 \pm 0.009$ & $0.050 \pm 0.005$ & $0.078 \pm 0.009$ \\
\hline 3868 [Ne III] & $0.418 \pm 0.021$ & $0.436 \pm 0.024$ & $0.398 \pm 0.023$ & $0.423 \pm 0.026$ \\
\hline $3889 \mathrm{He} \mathrm{I} \mathrm{+} \mathrm{H8}$ & $0.147 \pm 0.011$ & $0.181 \pm 0.015$ & $0.145 \pm 0.012$ & $0.178 \pm 0.016$ \\
\hline $3967[\mathrm{Ne}$ III $]+\mathrm{H} 7$ & $0.269 \pm 0.013$ & $0.304 \pm 0.016$ & $0.257 \pm 0.014$ & $0.295 \pm 0.017$ \\
\hline $4026 \mathrm{He} \mathrm{I}$ & $0.018 \pm 0.003$ & $0.019 \pm 0.003$ & $0.018 \pm 0.003$ & $0.019 \pm 0.003$ \\
\hline 4069 [S II] & $0.010 \pm 0.003$ & $0.010 \pm 0.003$ & $0.012 \pm 0.003$ & $0.013 \pm 0.003$ \\
\hline $4101 \mathrm{H} \delta$ & $0.230 \pm 0.011$ & $0.260 \pm 0.013$ & $0.221 \pm 0.012$ & $0.255 \pm 0.015$ \\
\hline $4340 \mathrm{H} \gamma$ & $0.466 \pm 0.021$ & $0.490 \pm 0.023$ & $0.449 \pm 0.023$ & $0.480 \pm 0.026$ \\
\hline 4363 [O III] & $0.171 \pm 0.008$ & $0.172 \pm 0.009$ & $0.163 \pm 0.010$ & $0.166 \pm 0.010$ \\
\hline $4471 \mathrm{He} \mathrm{I}$ & $0.047 \pm 0.004$ & $0.047 \pm 0.004$ & $0.037 \pm 0.003$ & $0.037 \pm 0.003$ \\
\hline 4658 [Fe III] & $0.004 \pm 0.002$ & $0.004 \pm 0.002$ & $0.003 \pm 0.001$ & $0.003 \pm 0.001$ \\
\hline 4686 He II & $0.019 \pm 0.002$ & $0.019 \pm 0.002$ & $0.019 \pm 0.002$ & $0.019 \pm 0.002$ \\
\hline 4701 [Fe III] & $0.001 \pm 0.001$ & $0.001 \pm 0.001$ & $0.004 \pm 0.002$ & $0.004 \pm 0.002$ \\
\hline 4713 [Ar IV] + He I & $0.023 \pm 0.002$ & $0.023 \pm 0.002$ & $0.022 \pm 0.002$ & $0.022 \pm 0.002$ \\
\hline 4740 [Ar IV] & $0.015 \pm 0.002$ & $0.015 \pm 0.002$ & $0.015 \pm 0.002$ & $0.015 \pm 0.002$ \\
\hline $4861 \mathrm{H} \beta$ & $1.000 \pm 0.045$ & $1.000 \pm 0.047$ & $1.000 \pm 0.058$ & $1.000 \pm 0.059$ \\
\hline 4907 [Fe IV] & $0.006 \pm 0.002$ & $0.006 \pm 0.002$ & $0.007 \pm 0.003$ & $0.007 \pm 0.003$ \\
\hline $4922 \mathrm{He} \mathrm{I}$ & $0.012 \pm 0.002$ & $0.011 \pm 0.002$ & $0.014 \pm 0.003$ & $0.013 \pm 0.003$ \\
\hline 4959 [O III] & $2.029 \pm 0.089$ & $1.950 \pm 0.089$ & $1.966 \pm 0.099$ & $1.923 \pm 0.099$ \\
\hline 4989 [Fe III] & $0.005 \pm 0.002$ & $0.005 \pm 0.002$ & $0.007 \pm 0.003$ & $0.006 \pm 0.003$ \\
\hline 5007 [O III] & $6.089 \pm 0.268$ & $5.834 \pm 0.266$ & $5.900 \pm 0.300$ & $5.751 \pm 0.298$ \\
\hline 5041 [Fe IV] & $0.007 \pm 0.002$ & $0.006 \pm 0.002$ & $0.010 \pm 0.003$ & $0.010 \pm 0.003$ \\
\hline $5047 \mathrm{He} \mathrm{I}$ & $0.004 \pm 0.002$ & $0.004 \pm 0.002$ & $0.003 \pm 0.003$ & $0.003 \pm 0.003$ \\
\hline 5158 [Fe II] & $0.006 \pm 0.001$ & $0.005 \pm 0.001$ & $0.004 \pm 0.002$ & $0.004 \pm 0.002$ \\
\hline 5199 [N I] & $0.006 \pm 0.002$ & $0.005 \pm 0.002$ & $0.005 \pm 0.002$ & $0.005 \pm 0.002$ \\
\hline 5271 [Fe III $]$ & $0.009 \pm 0.003$ & $0.008 \pm 0.003$ & $0.012 \pm 0.003$ & $0.011 \pm 0.003$ \\
\hline 5518 [Cl III] & $0.004 \pm 0.001$ & $0.004 \pm 0.001$ & $\ldots$ & $\ldots$ \\
\hline 5538 [Cl III] & $0.003 \pm 0.002$ & $0.003 \pm 0.002$ & $\ldots$ & $\ldots$ \\
\hline 5755 [N II] & $0.009 \pm 0.003$ & $0.009 \pm 0.002$ & $\ldots$ & $\ldots$ \\
\hline $5876 \mathrm{He}$ I & $0.140 \pm 0.009$ & $0.127 \pm 0.009$ & $0.140 \pm 0.008$ & $0.130 \pm 0.008$ \\
\hline 6300 [O I] & $0.024 \pm 0.004$ & $0.022 \pm 0.004$ & $0.021 \pm 0.004$ & $0.019 \pm 0.004$ \\
\hline 6312 [S III] & $0.011 \pm 0.004$ & $0.010 \pm 0.004$ & $0.016 \pm 0.004$ & $0.014 \pm 0.003$ \\
\hline 6364 [O I] & $0.008 \pm 0.002$ & $0.007 \pm 0.002$ & $0.006 \pm 0.002$ & $0.005 \pm 0.002$ \\
\hline 6548 [N II] & $0.027 \pm 0.002$ & $0.024 \pm 0.002$ & $0.027 \pm 0.003$ & $0.024 \pm 0.003$ \\
\hline $6563 \mathrm{H} \alpha$ & $3.126 \pm 0.139$ & $2.756 \pm 0.137$ & $3.075 \pm 0.156$ & $2.758 \pm 0.154$ \\
\hline 6584 [N II] & $0.081 \pm 0.012$ & $0.071 \pm 0.011$ & $0.080 \pm 0.011$ & $0.072 \pm 0.010$ \\
\hline $6678 \mathrm{He} \mathrm{I}$ & $0.035 \pm 0.003$ & $0.031 \pm 0.003$ & $0.034 \pm 0.003$ & $0.030 \pm 0.003$ \\
\hline 6717 [S II] & $0.043 \pm 0.004$ & $0.038 \pm 0.003$ & $0.046 \pm 0.004$ & $0.041 \pm 0.004$ \\
\hline 6731 [S II] & $0.037 \pm 0.004$ & $0.032 \pm 0.003$ & $0.040 \pm 0.004$ & $0.035 \pm 0.004$ \\
\hline $7065 \mathrm{He} \mathrm{I}$ & $0.081 \pm 0.005$ & $0.070 \pm 0.005$ & $\ldots$ & $\ldots$ \\
\hline 7136 [Ar III] & $0.040 \pm 0.004$ & $0.034 \pm 0.003$ & $\ldots$ & $\ldots$ \\
\hline $\mathrm{C}(\mathrm{H} \beta) \operatorname{dex}$ & \multicolumn{2}{|c|}{$0.12 \pm 0.06$} & \multicolumn{2}{|c|}{$0.12 \pm 0.07$} \\
\hline$E W($ abs $) \AA$ & \multicolumn{2}{|c|}{$3.75 \pm 0.41$} & \multicolumn{2}{|c|}{$3.55 \pm 0.42$} \\
\hline$F(\mathrm{H} \beta)^{a}$ & \multicolumn{2}{|c|}{$258 \pm 3$} & \multicolumn{2}{|c|}{$313 \pm 9$} \\
\hline$E W(\mathrm{H} \beta) \AA$ & \multicolumn{2}{|c|}{$230 \pm 6$} & \multicolumn{2}{|c|}{$222 \pm 9$} \\
\hline
\end{tabular}

${ }^{a}$ In units of $10^{-16} \mathrm{ergs} \mathrm{s}^{-1} \mathrm{~cm}^{-2}$. 
Table 3. Abundances in the supergiant H II region.

\begin{tabular}{|c|c|c|}
\hline Value & MMT & BTA \\
\hline$T_{\mathrm{e}}(\mathrm{OIII})(\mathrm{K})$ & $18500 \pm 700$ & $18300 \pm 800$ \\
\hline$T_{\mathrm{e}}(\mathrm{OII})(\mathrm{K})$ & $15200 \pm 500$ & $15100 \pm 600$ \\
\hline$T_{\mathrm{e}}(\mathrm{SIII})(\mathrm{K})$ & $17000 \pm 500$ & $16900 \pm 600$ \\
\hline$N_{\mathrm{e}}(\mathrm{SII})\left(\mathrm{cm}^{-3}\right)$ & $300 \pm 300$ & $400 \pm 300$ \\
\hline $\mathrm{O}^{+} / \mathrm{H}^{+}\left(\times 10^{-5}\right)$ & $0.345 \pm 0.035$ & $0.357 \pm 0.042$ \\
\hline $\mathrm{O}^{++} / \mathrm{H}^{+}\left(\times 10^{-5}\right)$ & $3.941 \pm 0.355$ & $3.969 \pm 0.419$ \\
\hline $\mathrm{O}^{+++} / \mathrm{H}^{+}\left(\times 10^{-5}\right)$ & $0.082 \pm 0.014$ & $0.095 \pm 0.022$ \\
\hline $\mathrm{O} / \mathrm{H}\left(\times 10^{-5}\right)$ & $4.369 \pm 0.357$ & $4.421 \pm 0.422$ \\
\hline $12+\log (\mathrm{O} / \mathrm{H})$ & $7.64 \pm 0.04$ & $7.65 \pm 0.04$ \\
\hline $\mathrm{N}^{+} / \mathrm{H}^{+}\left(\times 10^{-7}\right)$ & $5.157 \pm 0.701$ & $5.265 \pm 0.660$ \\
\hline $\mathrm{ICF}(\mathrm{N})$ & 12.65 & 12.40 \\
\hline $\log (\mathrm{N} / \mathrm{O})$ & $-0.83 \pm 0.07$ & $-0.83 \pm 0.07$ \\
\hline $\mathrm{Ne}^{++} / \mathrm{H}^{+}\left(\times 10^{-5}\right)$ & $0.610 \pm 0.058$ & $0.606 \pm 0.068$ \\
\hline $\mathrm{ICF}(\mathrm{Ne})$ & 1.11 & 1.11 \\
\hline $\log (\mathrm{Ne} / \mathrm{O})$ & $-0.81 \pm 0.05$ & $-0.82 \pm 0.06$ \\
\hline $\mathrm{S}^{+} / \mathrm{H}^{+}\left(\times 10^{-7}\right)$ & $0.695 \pm 0.061$ & $0.767 \pm 0.074$ \\
\hline $\mathrm{S}^{++} / \mathrm{H}^{+}\left(\times 10^{-7}\right)$ & $3.437 \pm 1.294$ & $5.196 \pm 1.282$ \\
\hline $\operatorname{ICF}(S)$ & 2.91 & 2.87 \\
\hline $\log (\mathrm{S} / \mathrm{O})$ & $-1.56 \pm 0.14$ & $-1.41 \pm 0.10$ \\
\hline $\mathrm{Cl}^{++} / \mathrm{H}^{+}\left(\times 10^{-7}\right)$ & $0.121 \pm 0.004$ & $\ldots$ \\
\hline $\mathrm{ICF}(\mathrm{Cl})$ & 2.19 & $\ldots$ \\
\hline $\log (\mathrm{Cl} / \mathrm{O})$ & $-3.22 \pm 0.14$ & $\cdots$ \\
\hline $\mathrm{Ar}^{++} / \mathrm{H}^{+}\left(\times 10^{-7}\right)$ & $1.003 \pm 0.101$ & $\ldots$ \\
\hline $\mathrm{Ar}^{+++} / \mathrm{H}^{+}\left(\times 10^{-7}\right)$ & $1.402 \pm 0.193$ & $1.395 \pm 0.317$ \\
\hline $\mathrm{ICF}(\mathrm{Ar})$ & 1.01 & 1.71 \\
\hline $\log (\mathrm{Ar} / \mathrm{O})$ & $-2.26 \pm 0.05$ & $-2.27 \pm 0.11$ \\
\hline $\mathrm{Fe}^{++} / \mathrm{H}^{+}\left(\times 10^{-7}\right)$ & $0.663 \pm 0.321$ & $0.622 \pm 0.212$ \\
\hline $\mathrm{ICF}(\mathrm{Fe})$ & 15.79 & 15.45 \\
\hline $\log (\mathrm{Fe} / \mathrm{O})$ & $-1.62 \pm 0.21$ & $-1.66 \pm 0.15$ \\
\hline$[\mathrm{O} / \mathrm{Fe}]$ & $0.20 \pm 0.21$ & $0.24 \pm 0.15$ \\
\hline
\end{tabular}

is barely detected in our spectra. Its flux as estimated $(S / N$ ratio 2) from the MMT spectrum, is $2.4 \times 10^{-16} \mathrm{erg} \mathrm{cm}^{-2} \mathrm{~s}^{-1}$. The parameters of fitted lines in different spectra are consistent within their rather large uncertainties. The parameters of narrow and broad lines are presented in Tables 2 and 4, respectively.
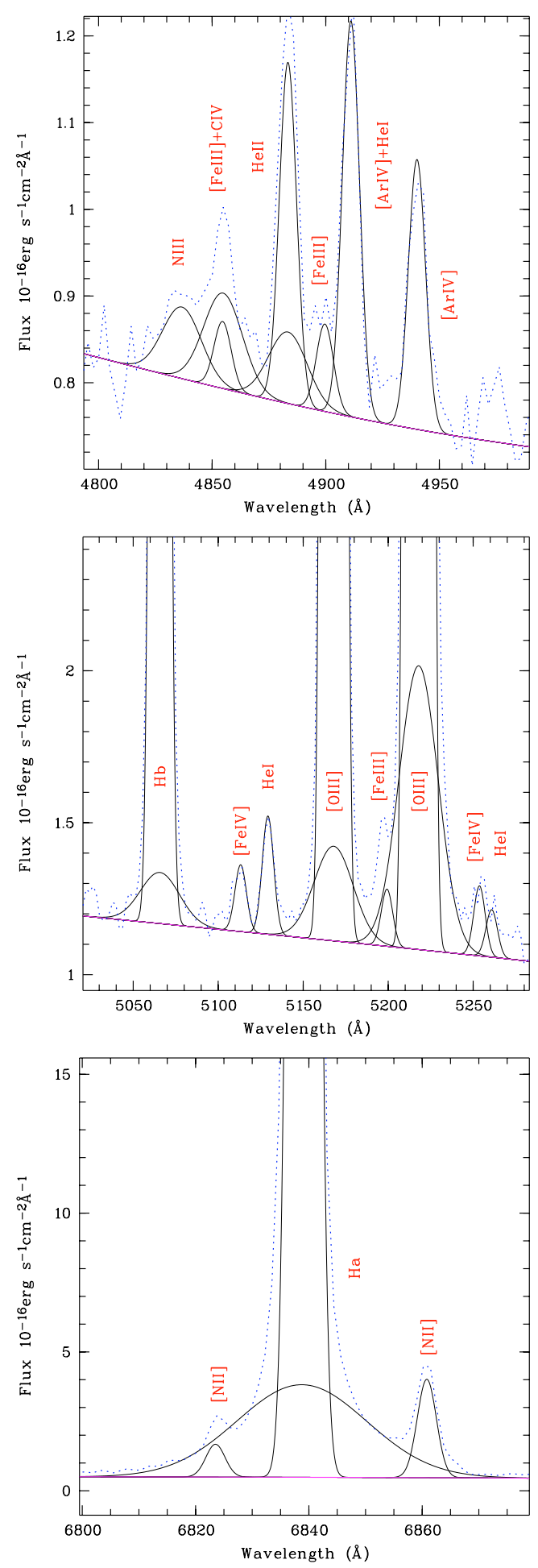

Fig. 3. Gaussian multicomponent fitting (solid lines) of the BTA spectra of HS $0837+4717$ (dotted lines). Top panel: region of the blue bump; middle panel: region of $\mathrm{H} \beta$ and [O III] $\lambda \lambda 4959,5007$; bottom panel: region of $\mathrm{H} \alpha,[\mathrm{N} \mathrm{II}] \lambda \lambda 6548,6583$.

\subsubsection{The broad components of the strong emission lines}

The broad components of the strong emission lines of $\mathrm{H} \alpha$, $\mathrm{H} \beta$ and [O III] $\lambda \lambda 4959,5007$ originate in the ionized gas moving with velocities of $\sim 1-2$ thousand $\mathrm{km} \mathrm{s}^{-1}$. Such high 


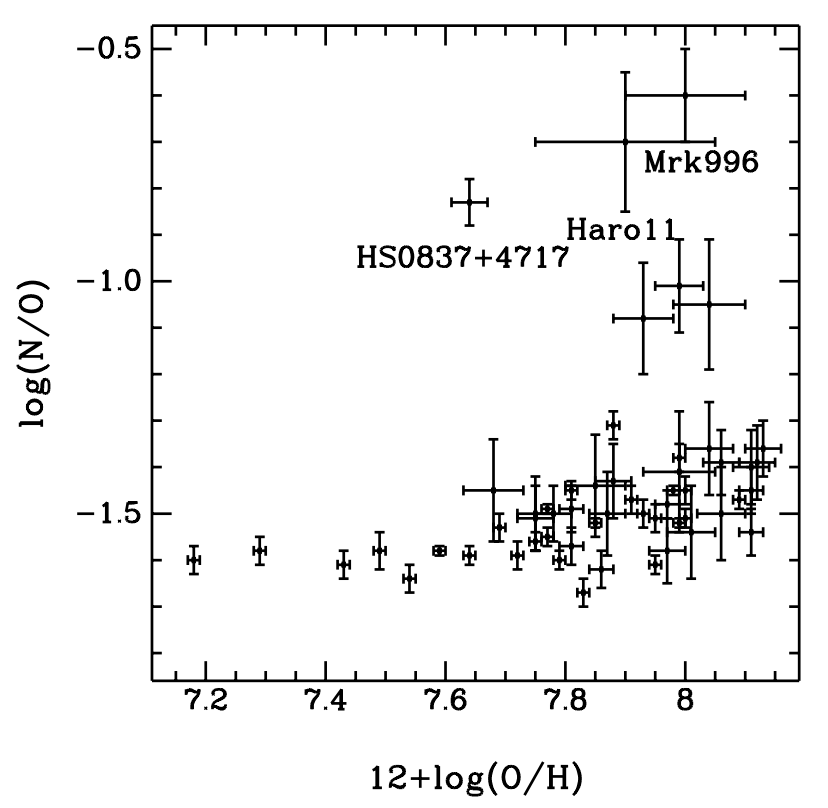

Fig. 4. Distribution of $\log (\mathrm{N} / \mathrm{O})$ ratio versus $12+\log (\mathrm{O} / \mathrm{H})$ for the BCG sample from Izotov \& Thuan (1999) with the value for HS 0837+4717 shown. Data for Haro 11 and Mkn 996 are from Bergvall \& Östlin (2002) and Thuan et al. (1996), respectively. The three galaxies with $\log (\mathrm{N} / \mathrm{O})$ in the range -1.1 to -1.0 are $\mathrm{UM} 420$, UM 448 and Mkn 1089.

velocities are related either to supernovae shells or to stellar winds of massive stars. Since the widths of the Balmer and the $[\mathrm{O} \mathrm{III}]$ lines are similar, it is reasonable to suggest that both originate in the same regions. While broad features centered at $\mathrm{H} \beta$ (the $\mathrm{He}$ II $\lambda 4861+\mathrm{H} \beta$ blend) and $\mathrm{H} \alpha$ (the He II $\lambda 6562+\mathrm{H} \alpha$ blend), characteristic of WR stars (e.g., SV98), should contribute to the broad components of the Balmer lines, their relative strengths calculated from models are expected to be tens of times lower than ones observed in HS 0837+4717. Therefore their origin in this galaxy, as well as in other similar cases, can probably be attributed to rather young remnants of multiple SN explosions. Having the line intensity ratios for the broad components, we can address the physical conditions in the regions of their formation. The parameters of broad components of these four lines are presented in Table 4. It is evident that these components have systematically higher line fluxes in the BTA spectra. This can be attributed to the wider slit (2" at BTA vs. 1".5 at MMT) and to the different PA.

The Balmer decrement of the broad line components, $I(\mathrm{H} \alpha) / I(\mathrm{H} \beta)$ appears unusually large. Accounting for the significant uncertainties, we accept the average of the MMT and BTA ratios for the fluxes of the broad and narrow components of $\mathrm{H} \beta$, equal to $(2.7 \pm 0.5) \%$. The same average ratio for $\mathrm{H} \alpha$ is $(19 \pm 1.5) \%$. Adopting a flux ratio of the narrow $\mathrm{H} \alpha$ to $\mathrm{H} \beta$ lines, corrected for extinction, of 2.76, a Balmer ratio for the broad components is $19.4 \pm 4$.

Such a large Balmer decrement is rather unusual. To ensure its reality we checked two possible factors, which could in principle enhance the Balmer ratio. The first one is underlying
Table 4. Parameters of the WR lines and of the broad components.

\begin{tabular}{|c|c|c|}
\hline & MMT & BTA \\
\hline \multicolumn{3}{|c|}{ Flux $\left(10^{-16} \mathrm{ergs} \mathrm{s}^{-1} \mathrm{~cm}^{-2}\right)^{a}$} \\
\hline 4640 N III (WR) & 2.5 & 3.8 \\
\hline 4658 C IV (WR) & 2.6 & 5.0 \\
\hline 4686 He II (WR) & 2.4 & 3.7 \\
\hline $4861 \mathrm{H} \beta$ & 6.3 & 15.0 \\
\hline 4959 [O III] & 11.6 & 18.3 \\
\hline 5007 [O III] & 34.8 & 55.0 \\
\hline $6563 \mathrm{H} \alpha$ & 159.4 & 261.3 \\
\hline \multicolumn{3}{|c|}{ Equivalent width $[\AA]$} \\
\hline 4640 N III (WR) & 1.4 & 2.1 \\
\hline 4658 C IV (WR) & 1.5 & 2.8 \\
\hline $4686 \mathrm{He}$ II (WR) & 1.4 & 2.1 \\
\hline $4861 \mathrm{H} \beta$ & 4.0 & 7.8 \\
\hline 4959 [O III] & 7.9 & 9.8 \\
\hline 5007 [O III] & 24.3 & 30.0 \\
\hline $6563 \mathrm{H} \alpha$ & 238.4 & 302.2 \\
\hline \multicolumn{3}{|c|}{$F W H M\left[\mathrm{~km} \mathrm{~s}^{-1}\right]$} \\
\hline 4640 N III (WR) & 1300 & 1300 \\
\hline 4658 C IV (WR) & 1300 & 1300 \\
\hline $4686 \mathrm{He}$ II (WR) & 1300 & 1300 \\
\hline $4861 \mathrm{H} \beta$ & 1500 & 1500 \\
\hline 4959 [O III] & 1500 & 1500 \\
\hline 5007 [O III $]$ & 1500 & 1500 \\
\hline $6563 \mathrm{H} \alpha$ & 1300 & 900 \\
\hline \multicolumn{3}{|c|}{ Broad/Nebular flux ratio [\%] } \\
\hline 4686 He II (WR) & 36 & 46 \\
\hline $4861 \mathrm{H} \beta$ & 1.9 & 3.5 \\
\hline 4959 [O III] & 1.7 & 2.2 \\
\hline 5007 [O III] & 1.7 & 2.2 \\
\hline $6563 \mathrm{H} \alpha$ & 16 & 22 \\
\hline \multicolumn{3}{|c|}{ WR/O stars ratio } \\
\hline Observed & 0.02 & \\
\hline Model $^{b}$ & 0.02 & \\
\hline
\end{tabular}

${ }^{a}$ Flux errors are $\sim 50 \%$ for the broad He II $\lambda 4686$ line. and $\sim(15-25) \%$ for the broad components of the strong lines.

${ }^{b}$ Data from Schaerer \& Vacca (1998).

stellar absorption in the Balmer lines, which has an $E W$ comparable to the $E W($ broad $\mathrm{H} \beta)$, and hence could affect it. However, since the $F W H M$ of stellar absorption lines $\left(\sim 200 \mathrm{~km} \mathrm{~s}^{-1}\right)$ is typically $\sim 7$ times smaller than that of broad emission component, the effect of stellar $\mathrm{H} \beta$ absorption will decrease the flux of broad emission $\mathrm{H} \beta$ at most by $15 \%$. The second factor could be purely instrumental: light scattering in the spectrograph of the strong lines could produce some artifacts with arbitrary ratio of 

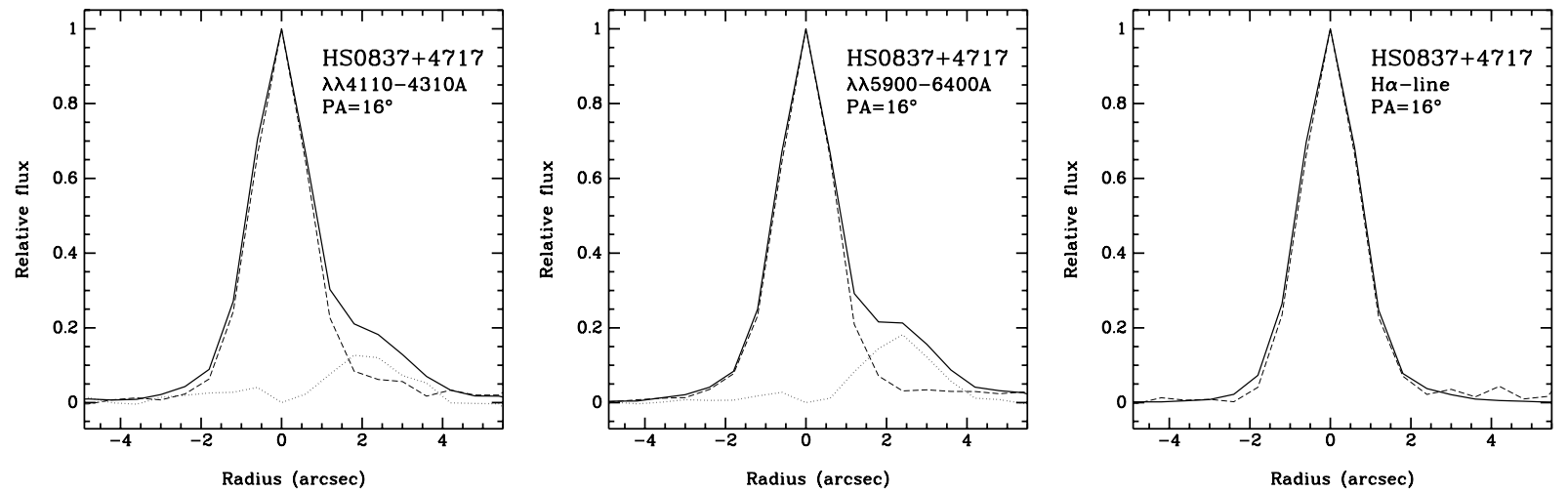

Fig. 5. Left panel: intensity distribution along the slit of the continuum between $\mathrm{H} \delta$ and $\mathrm{H} \gamma$. The dashed line shows the PSF (point-spread function) fitted to the central peak (related to the starburst). The dotted line shows the difference between the observed full light and that of the central bright compact region. The additional component, centered at $\approx 2$ '. 2 to the SSW is $\sim 0.25$ of the bright peak amplitude. Middle panel: same as in the left panel, but for continuum in the red. The relative flux of the fainter knot is 0.28 , that is a factor of 1.13 larger than in the blue. This implies that this knot in $(B-R)$ is $\sim 0.13$ redder than the central starburst, which mainly defines the integrated colours of the BCG. Right panel: same as the left panel, but for intensity centered on the $\mathrm{H} \alpha$ line. No significant signal is seen at the periphery besides the scattered light from the central bright region.

intensities. This certainly can be excluded, since we observed another object during the same night at the MMT and found even stronger emission lines, but with no hints of the broad components.

Thus, this very large Balmer decrement is either related to substantial internal extinction of the regions where the broad Balmer lines are formed, or to the excitation of hydrogen levels by electron collisions, or both. For the latter, $\mathrm{H} \alpha / \mathrm{H} \beta$ ratio can reach the value of 5.8 for $T_{\mathrm{e}}=10000 \mathrm{~K}$ (Chamberlain 1953). Thus, even for the case of electron excitation, some significant extinction (accounting for the additional factor of at least $\sim 3.3 \pm 0.7$ in the Balmer line ratio) should be invoked for the regions of the broad Balmer lines formation. Using the Whitford extinction law, the latter possibility corresponds to $E(B-V)=1.16 \pm 0.2$ and an additional extinction in the $B$-band of $A_{\mathrm{B}} \sim 5 \pm 1 \mathrm{mag}$ ! The latter indicates that the broad line emission forms in, or propagates through, a very dusty medium, contrary to what is observed for the narrow line $\mathrm{H}$ II region type emission. If no alternative explanation of the large Balmer decrement is found, this implies that the fluxes of broad features, and of the unknown optical continuum related to them, should be corrected by a factor of 25-100, depending on the wavelength. Such correction will raise the total luminosity of the BCG by a factor of several. The highly obscured regions are quite typical of starbursts observed in mergers. Moreover, in the XMD BCG SBS 0335-052 E mid-IR observations reveal a highly obscured starburst (Hunt et al. 2001).

We also point out the very large difference between the ratio of $I(\lambda 4959) / I(\mathrm{H} \beta)$ for the narrow and the broad components: for the average of the MMT and BTA data they are 1.88 and 0.62 , respectively. Is this due to the effect of significant collisional de-excitation of $\mathrm{O}^{++}$ions, or to a significantly lower $\mathrm{O} / \mathrm{H}$ ratio in the regions of broad line formation, or their lower $T_{\mathrm{e}}$ ? It is possible that all these factors combine to produce the observed effect. To answer these questions, higher $S / N$ ratio data will be helpful.

\subsection{Spatial structure of the emission regions}

Below we exploit the advantage of long-slit spectra to examine various morphological features of the object. The seeing during the MMT observations $(\theta=1 . \prime 6)$ and BTA observations in the red $\left(\theta=11^{\prime \prime} 3\right)$ was significantly less than the full extent of both line and continuum emission $\left(8^{\prime \prime}-10^{\prime \prime}\right)$, and allows the performance of a coarse analysis of the BCG substructure. Note that what we refer to as seeing is the width of an unresolved object as measured perpendicular to the spectrograph's dispersion and so includes contributions from the atmosphere as well as the telescope and spectrograph optics.

In Fig. 5 we show the intensity profiles of the continuum along the slit on the MMT spectrum averaged over the linefree spectral ranges (in the rest frame system) of 4110-4310 and 5900-6400 $\AA$. For comparison, the intensity distribution in a narrow band, centered on the $\mathrm{H} \alpha+[\mathrm{NII}]$ lines is also presented.

The intensity distributions of the continuum in Fig. 5 and of the strong emission lines are different. The latter have well fitted PSF profiles, with the $F W H M$ close to that of seeing. Some very faint wings/pedestal are present, which have just the appearance of the PSF at a few per cent level of the peak value.

The spatial distribution of continuum in the BCG shows some additional features. The main peak, related to the current starburst, fully coincides in position (along the slit) with the peak of the line emission. This starburst continuum component dominates the other components. However, an additional component (knot) on the MMT spectrum is seen at $\sim 2$ '. $2(1.8 \mathrm{kpc})$ to the SSW, with the relative flux of $\sim 25 \%$ in the blue and of $\sim 28 \%$ in the red. This implies that the $(B-R)$ of the faint "knot" is $\sim 0$. 13 redder than that of the current starburst, which dominates the BCG light and determines its integrated colours. Based on the relation above we can approximately estimate the $(B-R)$ colour of the faint knot. From the data in Table 5, the integrated $(B-R)_{0}$ is 0.15 . This colour is affected by the strong emission lines included in both filters. Accounting for 
Table 5. Main parameters of HS $0837+4717$.

\begin{tabular}{|c|c|}
\hline Parameter & Value \\
\hline $\mathrm{RA}(\mathrm{J} 2000.0)$ & 084029.84 \\
\hline $\operatorname{Dec}(\mathrm{J} 2000.0)$ & +470109.1 \\
\hline$A_{\mathrm{B}}^{(N)}$ & 0.11 \\
\hline$B^{\text {tot }}$ & $18.18 \pm 0.10^{(2)}$ \\
\hline$(U-B)_{0}^{\mathrm{tot}}$ & $-0.41 \pm 0.14^{(2)}$ \\
\hline$(B-V)_{0}^{\mathrm{tot}}$ & $0.86 \pm 0.14^{(1,2)}$ \\
\hline$(V-R)_{0}^{\mathrm{tot}}$ & $-0.71 \pm 0.14^{(1,2)}$ \\
\hline $\mathrm{V}_{\mathrm{Hel}}\left(\mathrm{km} \mathrm{s}^{-1}\right)$ & $12630^{(2)}$ \\
\hline Distance (Mpc) & $170.5^{(2)}$ \\
\hline$M_{\mathrm{B}}^{0(3)}$ & $-18.1^{(2)}$ \\
\hline Opt. size $\left({ }^{\prime \prime}\right)^{4}$ & $\sim 8 \times 7^{(2)}$ \\
\hline Opt. size (kpc) & $6.5 \times 6^{(2)}$ \\
\hline $12+\log (\mathrm{O} / \mathrm{H})$ & $7.64 \pm 0.03^{(2)}$ \\
\hline$T_{\mathrm{e}}(\mathrm{O}$ III $)(\mathrm{K})$ & $18000^{(2)}$ \\
\hline$N_{\mathrm{e}}\left(\mathrm{cm}^{-3}\right)$ & $\lessgtr 400^{(2)}$ \\
\hline $\log (\mathrm{N} / \mathrm{O})$ & $-0.83 \pm 0.05^{(2)}$ \\
\hline H I int.flux ${ }^{(5)}$ & $<0.31-0.43^{(2)}$ \\
\hline$M(\mathrm{H} \mathrm{I})\left(10^{9} M_{\odot}\right)$ & $<2.5^{(2)}$ \\
\hline$M(\mathrm{H} \mathrm{I}) / L_{\mathrm{B}}{ }^{(6)}$ & $<0.9^{(2)}$ \\
\hline
\end{tabular}

(1) $-(B-V)_{\text {tot }}^{0}$ corrected for redshift is 0.21 .

(1) $-(V-R)_{\text {tot }}^{0}$ corrected for redshift is -0.02 .

(2) - this paper.

(3) - corrected only for the Galactic extinction.

(4) $-a \times b$ at $\mu_{\mathrm{B}}=25 \mathrm{mag} / \square^{\prime \prime}$.

(5) - for $W_{50} \leq 100-140 \mathrm{~km} \mathrm{~s}^{-1}$, in Jy km s${ }^{-1}$.

(6) - in units $\left(M / L_{\mathrm{B}}\right)_{\odot}$.

$(N)$ - data from the NED.

the EWs of these lines and for the effective widths of both filters, we estimate that the $(B-R)$ of the continuum component is redder due to the emission lines by $\sim 0.12$. This implies that the net continuum of HS $0837+4717$ has a $(B-R)_{0} \sim 0.03$. The latter is very similar to the colour $(B-R)=-0.05$ of the mixture of a starburst with an age of 3.7 Myr and the continuum produced by gas at $T_{\mathrm{e}}=20000 \mathrm{~K}$. The former, according to the PEGASE. 2 model (with a Salpeter IMF with limits of 0.1 and $120 M_{\odot}$ and $\left.Z=Z_{\odot} / 20\right)$, is $(B-R)=-0.24$, while the latter, from the data in Aller $(1984)$, has $(B-R)=+0.53$. They are mixed in the proportion that results in the observed narrow-line $E W(\mathrm{H} \alpha) \sim 1400 \AA$. We accept the bright component continuum colour of $(B-R)=-0.05$, and from the latter we obtain for the faint knot $(B-R)_{0}^{\text {faint }} \sim 0.08$. For an instantaneous star formation (SF) with the same IMF and $Z=Z_{\odot} / 20$, this $(B-R)$ corresponds to an age of $\sim 25 \mathrm{Myr}$. Apart from this fainter knot of continuum emission, there is also some diffuse component, stretching further to the $\mathrm{SW}$ and $\mathrm{NE}$, as seen at the $V$-band image in Fig. 1.

We estimate the intrinsic size of the supergiant $\mathrm{H}$ II region from a comparison of the FWHM of its strong emission lines with that of PSF. Unfortunately, for the sharpest images $(\theta=1$ '. 3 , BTA observations in the red), the seeing fluctuated, therefore it is difficult to accept the value of the FWHM for the PSF. For the MMT observations the situation is better. A nearby star appears on the slit and can be used to estimate the PSF at various wavelengths. The weighted mean of the $F W H M(\mathrm{PSF})$ in red and green is $11^{\prime \prime} 60$. The FWHM(intrinsic), derived as the weighted mean of the values in red and green, is $\sim 0 \prime^{\prime} 7 \pm 0$. $^{\prime} 07$. FWHM(intrinsic) were calculated by quadratic subtraction of the $F W H M(\mathrm{PSF})$ from the measured $F W H M$ of strong lines. The size of the faint knot is difficult to measure directly. However, from the decomposition of the light distribution along the slit, shown in Fig. 5, the FWHM of this knot is $\sim 2$ '. 2. Adopting the same $F W H M$ of the PSF, we obtain a coarse estimate for the intrinsic size of the faint knot as $F W H M($ knot $) \sim 1$ '.5. These sizes correspond to a $F W H M$ linear diameters of the two components of $\sim 0.58 \mathrm{kpc}$ and $\sim 1.25 \mathrm{kpc}$, respectively.

Summarizing this analysis, we find that along the major axis at its brightest part, this BCG has a doublenucleus structure with characteristic sizes of the components of 0.6 and $1.25 \mathrm{kpc}$, respectively, and a projected distance of 2 .'2 (1.8 kpc) between the components. The total extent of the underlying low-surface-brightness (LSB) component is $\gtrsim 10^{\prime \prime}$ ( $8 \mathrm{kpc}$ ). The nature of the faint knot is not clear. But since it is also quite blue, one can expect that this is also related to the recent SF event.

\subsection{Ionized gas kinematics}

We performed an analysis of the gas kinematics following the method described by Zasov et al. (2000). The H $\alpha$ line positionvelocity $(\mathrm{P}-\mathrm{V})$ diagrams along the slit at $\mathrm{PA}=44^{\circ}$ and $130^{\circ}$ are shown in Fig. 6. The first one is close to the major axis of the outermost isophotes, while the second is nearly perpendicular. The full extent of $\mathrm{H} \alpha$ emission as seen from these spectra is the same in both directions: $9^{\prime \prime}-10^{\prime \prime}$ (or $\sim 7-8 \mathrm{kpc}$ ). The appearance of $\mathrm{P}-\mathrm{V}$ diagrams, however, is very different. For $\mathrm{PA}=130^{\circ}$ the radial velocity is practically constant for a range from $-3^{\prime \prime}$ to $\sim 3^{\prime \prime} .5$, or about $65 \%$ of the entire extent of the observed $\mathrm{H} \alpha$-emission. This is consistent with a negligible expected projection of the rotation velocity for this PA. The velocity curve at the galaxy edges looks, however, somewhat disturbed. This could be a result of a recent interaction or some indication for a gas blowout, confined in some directions. Better $S / N$ ratio data for these regions are necessary to confirm the significance of the velocity disturbances.

The slit position at $\mathrm{PA}=44^{\circ}$ is expected to be the optimal one to see the overall rotation of this galaxy. However, the respective $\mathrm{P}-\mathrm{V}$ diagram does not look like a typical rotation. We discuss below two possible explanations for the unusual distribution of the radial velocity.

The first one treats the $\mathrm{P}-\mathrm{V}$ diagram as the consequence of counter-rotation. At positive coordinates along the slit we see the receding branch of the rotation curve with a velocity amplitude of $50-70 \mathrm{~km} \mathrm{~s}^{-1}$ (depending on how the real curve is drawn between the two last points) relative to the brightness peak velocity. However, the symmetric (approaching) continuation of this branch at negative coordinates is not seen. Instead, the receding branch is present, with an 

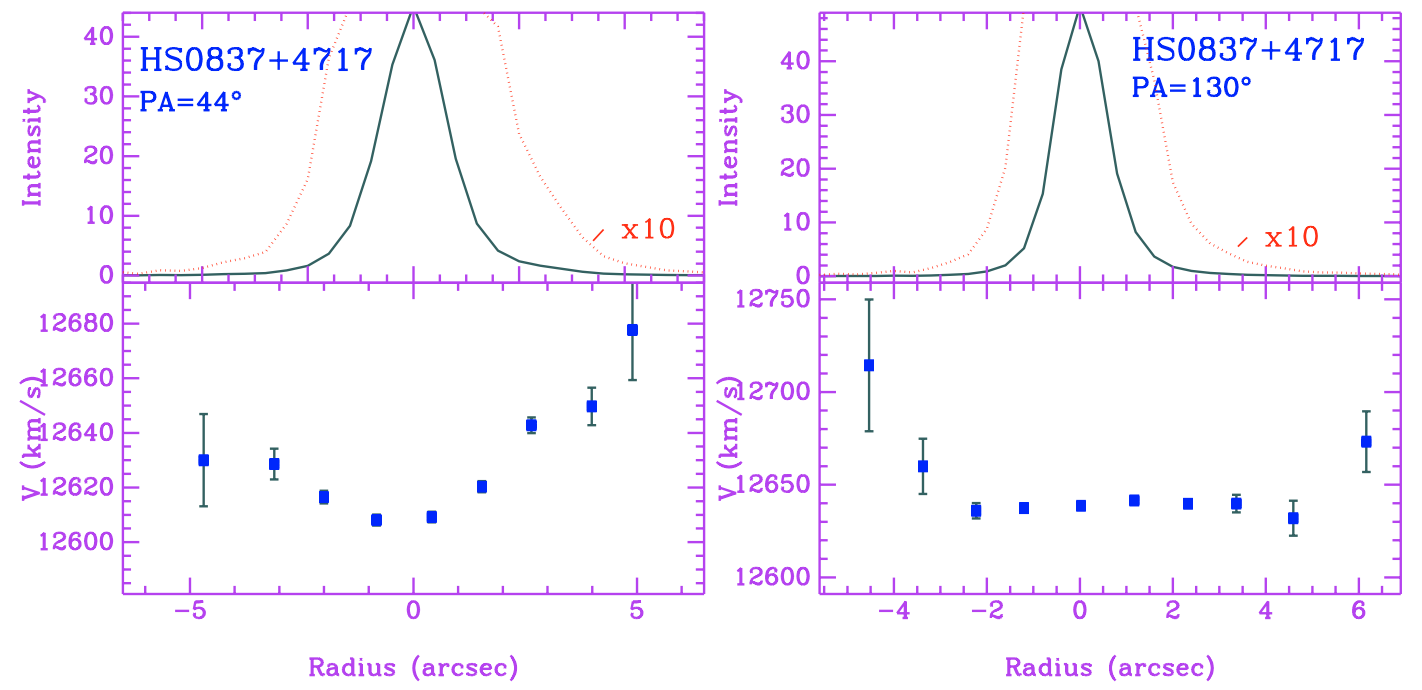

Fig. 6. Left panel: intensity distribution of $\mathrm{H} \alpha$ along the slit at $\mathrm{PA}=44^{\circ}$ and the respective $\mathrm{P}-\mathrm{V}$ diagram (binned with 3 pixels, or $1^{\prime \prime} .2$, corresponding to the seeing). The radial velocity increases monotonously in the SW direction, resembling the receding branch of rotation curve with the amplitude of 50-70 $\mathrm{km} \mathrm{s}^{-1}$. However, the expected approaching branch for negative coordinates in the direction to NE is absent (see text for discussion). Right panel: same as in the left panel but at $\mathrm{PA}=130^{\circ}$. The velocity variations in the central part of this cross-section are significantly smaller than those at $\mathrm{PA}=44^{\circ}$. This would be expected due to the small projection of the rotation velocity onto the minor axis. There seems to be a large velocity disturbance at the NW edge, where radial velocity steeply raises to $60-70( \pm 30) \mathrm{km} \mathrm{s}^{-1}$ in the two last points. The small velocity variations are also observed on the opposite side of the galaxy.

amplitude of $20-30 \mathrm{~km} \mathrm{~s}^{-1}$. This could probably indicate $2 \mathrm{dy}-$ namically decoupled gas systems. A difference of $\sim 30 \mathrm{~km} \mathrm{~s}^{-1}$ between the radial velocities near the peak of the $\mathrm{H} \alpha$ emission for $\mathrm{PA}=130^{\circ}$ and $\mathrm{PA}=44^{\circ}$ can be attributed to small difference in the zero-points of the dispersion curves for the two independent spectra.

Another explanation treats the part of $\mathrm{P}-\mathrm{V}$ diagram at negative coordinates as a significant velocity disturbance relative to the extrapolated approaching branch. This could be related to a strong outflow from the recent SF episode. If one is to draw the approaching branch of the rotation curve as a symmetric extrapolation of the receding one, the resulting difference between the observed data and drawn approaching branch shows us the form of the disturbance. This resembles the common wave-like form of supershells, as they are usually seen on $\mathrm{P}-\mathrm{V}$ diagrams of starbursting galaxies. The velocity of such supershell relative to the background rotation velocity is $\sim 70-100 \mathrm{~km} \mathrm{~s}^{-1}$. Its visible extent is $\sim 2^{\prime \prime}$. The results could indicate that only a half of the shell is seen, which is closer to the center. Therefore, its full extent could be $\sim 4^{\prime \prime}$, or $\sim 3.3 \mathrm{kpc}$. Such features in $\mathrm{P}-\mathrm{V}$ diagrams are detected in many BCGs, and the most plausible explanation is related to the expansion of supershells formed in the ionized gas due to the recent starforming activity in these objects. Supershells are produced by hot bubbles, originating from the energy injection of numerous massive star winds and supernovae ( $\mathrm{SNe}$ ) explosions into the galactic ISM (e.g., Tenorio-Tagle \& Bodenheimer 1988).

In particular, a similar, but scaled down according to the parameters of the host galaxy, supershell in HS $0822+3542$ was discussed by Pustilnik et al. (2003a). The energy of that supershell with a diameter of $0.48 \mathrm{kpc}$ and a velocity amplitude of $30 \mathrm{~km} \mathrm{~s}^{-1}$ was provided by $13 \mathrm{SNe}$. Assuming the same density of ambient gas of $0.1 \mathrm{~cm}^{-2}$, with the supershell parameters as observed in HS $0837+4717$ (adopting a minimal $V_{\text {shell }}=$ $70 \mathrm{~km} \mathrm{~s}^{-1}$ ), we derive a total energy larger by a factor of $\sim 1770$. This corresponds to $\sim 23000 \mathrm{SNe}$ exploded during a period of the order of one dynamical age of the supershell. The latter is $t_{\text {dyn }}=0.6 R_{\text {shell }} / V_{\text {shell }}=13.7 \mathrm{Myr}$ (Weaver et al. 1977). This would imply the existence of a star cluster with an age of $\sim 17 \mathrm{Myr}$, provided the mean rate $\sim 1700$ SNe per Myr during last $\sim 14 \mathrm{Myr}$ (recall, that $\mathrm{SNe}$ begin to explode in the cluster after $\sim 3 \mathrm{Myr}$ ). From this SN rate and a PEGASE.2 model with the same IMF as above, we derive the mass of such a cluster of $\sim 7.6 \times 10^{6} M_{\odot}$.

For a galaxy of this high luminosity and an apparent diameter of $\sim 10 \mathrm{kpc}$, the estimated rotation velocity is rather small. The inclination correction, if applicable, is modest. From the apparent axial ratio of this BCG ( $p=b / a=0.67)$, it follows, that in the case of a stable rotating disk, $i$ is quite large. Indeed, from the well known formula: $\cos ^{2}(i)=\left(p^{2}-q^{2}\right) /\left(1-q^{2}\right)$, and the range of intrinsic ratio for disk-like galaxies of $q=$ $b / a=0.2-0.3$, the $i$ would fall in the range of $49^{\circ}-52^{\circ}$. The respective correction of $1 / \sin (i)=1.27-1.32$. In this case the corrected $V_{\text {rot }}$ can reach the value of 65 to $90 \mathrm{~km} \mathrm{~s}^{-1}$, within the range characteristic of low-mass galaxies.

If this object is a merger, gas motions due to dissipative collisions could be significantly decoupled from the motion of stars. Until the system relaxes, the gas motions are bad tracers of mass distribution (e.g., Amram \& Östlin 2001). Therefore the amplitude of the rotation velocity observed in $\mathrm{H} \alpha$ could be unrelated to the overall gravitational potential.

\subsection{Imaging and photometry}

In the light of the discussion on the possible merger nature of HS $0837+4717$, it is important to inspect the 2D morphology 
of the galaxy. Its grey-scale $V$-band image with isophotes superimposed is shown in Fig. 1.

The overall structure is asymmetric and somewhat disturbed, both near the center, and in the peripheral regions. In particular, the fainter component at $\sim 2$ " to the South (which is seen in the continuum on the long-slit spectrum) is apparent on this image, however smeared due to the seeing effect. The outermost isophotes indicate several appendages to the main body. Disregarding the faintest isophote as being subject to noise, we notice two small features, at NNW and SSE of the center, the latter being much more prominent. The BCG images available from the Data Release 1 (DR1) database of the Sloan Digital Sky Survey (SDSS) (York et al. 2000; Abazajian et al. 2003) corroborate this disturbed morphology.

As described above, small transparency variations took place during imaging with the Wise 1-m telescope. In order to minimize their effect on the object's integrated magnitudes, we selected from each of the nights the brightest value in each of the bands, and compared them with the magnitudes, derived through the convolution of the MMT spectrum. For $U$-band we could not use an MMT measurement due to the limited wavelength coverage. Therefore, even though the $U$-band magnitudes for both nights are consistent within the observational uncertainties, they should be treated with caution. The brightest values for the Wise measurements in $B$ and $R$ are consistent with the MMT data within the uncertainties, therefore we accept them with an rms error of 0.10 . In the $V$-band, however, the brightest magnitude on Wise data is still 0.27 fainter than the respective magnitude derived from the MMT spectrum. Therefore, for $V$ we accept the MMT derived value. The integrated photometry data are summarized in Table 5.

The very red $(B-V)(\sim 0.9)$ and blue $(V-R)(\sim-0.7)$ colours of this object are related to the very large $E W$ s of the [O III] $\lambda 4959$ and $\lambda 5007 \AA$ emission lines, redshifted to the center of $V$-band. This can be checked by the convolution of the deredshifted BCG spectrum (see Table 5).

\subsection{HI results}

Effectively, we observed HS $0837+4717$ in "on+off" positions for $320 \mathrm{~min}$ and, after smoothing, obtained an rms noise of $1.3 \mathrm{mK}$. This translates to an rms in flux density of $1.6 \mathrm{mJy}$. Since no signal was detected, we put a $2 \sigma$ upper limit of $3.2 \mathrm{mJy}$ on the peak flux density from the galaxy. The full amplitude of ionized gas velocity variations, $\sim 100-140 \mathrm{~km} \mathrm{~s}^{-1}$ is used to estimate the upper limit of the H I flux. Adopting this value as an estimate of $W_{50}$ for the HI profile, the upper limit of the integrated H I flux is of $(0.32-0.45) \mathrm{Jy} \mathrm{km} \mathrm{s}^{-1}$. The respective upper limit on the H I mass is $M(\mathrm{HI}, \mathrm{BCG}) \leq$ $(2.1-3.0) \times 10^{9} M_{\odot}$.

\section{Discussion and conclusions}

\subsection{General comments}

The integrated parameters of HS $0837+4717$ are summarized in Table 5. There are two unusual properties, which are probably related. The first one is the high luminosity of this BCG for its very low metallicity (see, e.g., plots of $\mathrm{O} / \mathrm{H}$ versus $M_{\mathrm{B}}$ in Kunth \& Östlin 2000; Pustilnik et al. 2003b). The second one is the very large nitrogen abundance excess: $\mathrm{N} / \mathrm{O}$ is a factor of $\sim 6$ larger than for other BCGs with similar $\mathrm{O} / \mathrm{H}$. The first property can be attributed to an exceptionally strong starburst, which causes an additional brightening by several magnitudes. Pustilnik et al. (2001b) argued that the majority of starbursts in BCGs are triggered by interactions of various strengths with other galaxies. The range spreads from weak tidal interactions through strong tidals and the sinking of satellites to merging of low-mass galaxies. The latter case can be considered as a down-scaled analog of ultraluminous IR galaxies, mergers of massive gas-rich galaxies (e.g., Genzel et al. 2001). Below we discuss the possibility that HS $0837+4717$ as well could be an advanced merger.

\subsection{Possible evidences of merger}

Telles \& Terlevich (1997) found that the most luminous BCGs show disturbed external morphologies, while low luminosity BCGs show regular morphology. In their detailed studies of several luminous BCGs, Östlin et al. $(1999,2001)$ found morphological and kinematic evidences for the merger nature of these galaxies. HS $0837+4717$ is similar to their BCGs in several aspects.

As shown above, the overall disturbed morphology of this BCG, its "double-nucleus" appearance, and the presence of appendages on the periphery, presumably imply some recent disturbance. However, no disturbing galaxies are visible in the environment of this BCG (see Sect. 4.5).

In addition, the distribution of the ionized gas velocity also appears disturbed, mainly near the edges of the galaxy. The gas motions along the apparent major axis show large deviations from the expected regular rotation. These could be related either to a counter-rotating gaseous component, or to a very energetic supershell. The two knots, related to the current/recent starbursts are very massive. Indeed, from the photometry in Table 5 and numbers derived in Sect. 3.3, from a PEGASE. 2 model with $Z=Z_{\odot} / 20$, and a Salpeter IMF as above, we find the following. For the brighter component with $M_{\mathrm{B}}=-17.9$ and an age of $3.7 \mathrm{Myr}$ the mass of starburst is $0.8 \times 10^{7} M_{\odot}$. As shown above, a significant part of this starburst could be highly obscured by dust. Therefore, its unobscured blue luminosity could be several times higher than that listed here. This would translate to a total mass for this starburst region of $\gtrsim 3 \times 10^{7} M_{\odot}$. For the faint knot $M_{\mathrm{B} \text {,faint }}=-16.4$. Its colour $(B-R)$ corresponds to an instantaneous SF burst with an age of 25 Myr. With the same IMF as above, PEGASE.2 predicts a star cluster mass of $2.7 \times 10^{7} M_{\odot}$. Also, from the parameters of the suggested supershell at the NE edge of the galaxy, we estimate the mass of the embedded star cluster with an age of $\sim 17 \mathrm{Myr}$ as $\sim 0.76 \times 10^{7} M_{\odot}$. Such massive starbursts should involve molecular clouds with the masses of $10^{8} M_{\odot}$ or more. We will return to this point in Sect. 4.4. Finally, as shown, e.g., by Mihos \& Hernquist (1996), very energetic starbursts are characteristic of merging galaxies. 
An alternative option for such a case is a strong interaction with an undetected massive object, such as very LSB galaxy or a hydrogen cloud devoid of stars, similar to the case of Dw 1225+0152 (Salzer et al. 1991).

\subsection{WR star population}

The WR stellar population is observed only in four of about 30 XMD BCGs known. They include I Zw 18 (Izotov et al. 1997c; Legrand et al. 1997), SBS 0335-052E (Izotov et al. 2001), Tol 1214-277 (Fricke et al. 2001) and UM 133 (Kniazev et al. 2001, 2004, in prep.). Thus, HS $0837+4717$ is the fifth XMD BCG with a detected WR population. While the accuracy of the flux measurements of the broad WR features on the available spectra is far from sufficient for accurate prediction, it is useful to obtain an estimate of the WR-to-O star relative numbers.

To derive $N(\mathrm{WR}) / N(\mathrm{O}+\mathrm{WR})$, we follow the approach described in detail by Guseva et al. (2000). The number of WR stars was derived from the luminosity of the WR lines. Their fluxes were derived directly from the 1-D spectra, as discussed in Sect. 3.2.1. No aperture correction was applied to the WR-line fluxes, assuming that this emission is produced in a compact region with an angular size less than the width of the slit. The number of $\mathrm{O}$ stars was derived from the total $\mathrm{H} \beta$ luminosity for the brightest SF region $\left(L_{\text {cor }}\right)$, corrected for the contribution of WR stars and for the aperture effect. For the latter we adopt the circular symmetry of the bright SF region as seen in the direct image. To estimate the fraction of the undetected $\mathrm{H} \beta$ flux, the radial distribution of the $\mathrm{H} \beta$ line intensity, shown in Fig. 2, was used. The respective correction factor for the total $\mathrm{H} \beta$ flux is 1.66 .

The number of $\mathrm{O}$ stars is derived from the number of ionizing photons $Q_{0}^{\text {cor }}$, which is related to the total luminosity of the $\mathrm{H} \beta$ emission line $L_{\text {cor }}(\mathrm{H} \beta)$ by

$L_{\text {cor }}(\mathrm{H} \beta)=4.76 \times 10^{-13} Q_{0}^{\text {cor }}$.

For a representative O7V star we adopt the number of Lyman continuum photons emitted to be $Q_{0}^{\mathrm{O} 7 \mathrm{~V}}=1 \times 10^{49} \mathrm{~s}^{-1}$ (Leitherer 1990). The total number of $O$ stars is then derived from the number of O7V stars by correcting for other O star subtypes, using the parameter $\eta_{0}$ introduced by Vacca \& Conti (1992) and Vacca (1994). $\eta_{0}$ was calculated in SV98 as a function of the time elapsed since the onset of an instantaneous star formation burst, as inferred from the $E W(\mathrm{H} \beta)$. The Salpeter IMF with the $M_{\text {low }}$ and $M_{\text {up }}$ limits of $0.8 M_{\odot}$ and $120 M_{\odot}$ was adopted here. We assumed that the average Lyman continuum flux per one WR star $Q_{0}^{\mathrm{WR}}$ is comparable to $Q_{0}^{\mathrm{O} 7 \mathrm{~V}}$ and is equal to $1.0 \times$ $10^{49} \mathrm{~s}^{-1}$ (Schaerer et al. 1999). Finally

$N(\mathrm{O})=\frac{Q_{0}^{\mathrm{cor}}-N_{\mathrm{WR}} Q_{0}^{\mathrm{WR}}}{\eta_{0}(t) Q_{0}^{\mathrm{O} 7 \mathrm{~V}}}$.

In Sect. 3.2.1 we performed the estimates of the observed fluxes of all broad WR lines in the blue and red bumps (see Table 4). The latter are used to get the luminosities in the respective lines. Then, the luminosity of red bump is used to estimate the number of representative WC4 stars, having
$L_{5808}=3.0 \times 10^{36} \mathrm{erg} \mathrm{s}^{-1}: N_{\mathrm{WC}}=280$. The number of representative WN7 stars can be estimated either from its adopted luminosity in broad He II line, or from the sum luminosity of the blue bump, subtracting the contribution of C IV $\lambda 4658$, caused by WC stars. The uncertainties of all WR broad features are large, about $50 \%$. Therefore the derived numbers of WR stars are only indicative. Better signal-to-noise spectra are needed to study the WR population in this BCG at the quantitative level.

Assuming the luminosity of a single WN7 star to be $1.6 \times$ $10^{36} \mathrm{ergs} \mathrm{s}^{-1}$ in the broad He II $\lambda 4686$ line or $1.99 \times 10^{36} \mathrm{ergs} \mathrm{s}^{-1}$ in the blue bump (SV98), we estimate from the WR line fluxes derived on the MMT spectrum, $N_{\mathrm{WN}}$ to be either 500 , or 860 . Adopting the average of the two estimates for WN stars, we get the sum number of WR stars (WN+WC) in the young starburst to be $\sim 1000$.

Following to SV98 and using the observed value of $E W(\mathrm{H} \beta)=230 \AA$, we estimate the burst age and the parameter $\eta_{0}$ (updated model version of 1999, as presented in Starburst99) in HS 0837+4717 as $3.7 \mathrm{Myr}$, and $~ 0.65$, respectively. The total number of O stars in HS $0837+4717$ is found to be $\mathrm{N}(\mathrm{O}) \approx 47000$. This yields a relative number of WR stars to $\mathrm{O}$ stars $N(\mathrm{WR}) /(N(\mathrm{O}+\mathrm{WR})$ of 0.02 , coinciding with the model prediction in SV98.

\subsection{A large excess of the nitrogen abundance}

The possibility of enhanced $N$ abundance in galaxies with the spectral signatures of WR stars, in comparison to other galaxies of similar metallicity was first noticed by Pagel et al. (1986) where no details on the strength of this effect were given. The occurrences of a significant nitrogen excess in galaxies are very rare. For example, among more than one hundred BCGs with well measured heavy element abundances (e.g., IT99; Ugryumov et al. 2003) only four galaxies (Mkn 996, UM 420, Mkn 1089 and UM 448) exhibit a nitrogen excess of a factor of 3 and larger, relative to the value appropriate for their $\mathrm{O} / \mathrm{H}$ (Thuan et al. 1996; IT99). All four have $12+\log (\mathrm{O} / \mathrm{H}) \gtrsim 7.9$ and show broad WR lines (Guseva et al. 2000; Thuan et al. 1996). One more similar galaxy, Haro 11 is found among the luminous BCGs studied by Bergvall \& Östlin (2002). With $12+$ $\log (\mathrm{O} / \mathrm{H})=7.9$ it has a nitrogen abundance excess of a factor of $\sim 6$. Strong signatures of WR population are detected in this BCG as well.

An excess of the nitrogen abundance by a factor of 3 is also detected in two central $\mathrm{H}$ II regions (both with detectable WR population) of the nearby starbursting dwarf irregular galaxy NGC 5253 with $12+\log (\mathrm{O} / \mathrm{H})=8.16$ (Kobulnicky et al. 1997, and references therein). In Table 6 we summarize the parameters of all known such galaxies, and below draw some tentative empirical correlations. First, most of these galaxies could be identified as various stages of mergers. Second, as a subgroup of starbursting galaxies, $70 \%$ of them are luminous $\left(M_{\mathrm{B}}^{0}<-18^{\mathrm{m}} 0\right)$, thus are not classical dwarf galaxies. Third, all these objects are WR galaxies. Fourth, all these BCGs but one have $12+\log (\mathrm{O} / \mathrm{H})$ in the range of $\sim 7.9$ to 8.16 . HS $0837+4717$ is the only such galaxy belonging to the XMD group. 
Table 6. Starburst galaxies with significant nitrogen excess.

\begin{tabular}{llrllcll}
\hline \hline IAU Name & Synonyms & $V_{\mathrm{hel}}{ }^{a}$ & $B_{\mathrm{tot}}{ }^{a}$ & \multicolumn{1}{c}{$M_{B}$} & $12+\log (\mathrm{O} / \mathrm{H})$ & $\Delta \log (\mathrm{N} / \mathrm{O})$ & Notes \\
\hline $0034-3349$ & Haro11 & 6175 & 14.6 & -20.0 & 7.90 & 0.8 & merger \\
$0125-061$ & MKN996 & 1622 & 15.1 & -17.1 & 8.00 & $0.6-1.4$ & probable merger \\
$0218+003$ & UM420 & 17514 & 16.5 & -20.3 & 7.89 & 0.5 & sinking merger? \\
$0459-043$ & MKN1089 & 4107 & $15.0(13.3)$ & $-19.1(-20.8)$ & 8.07 & 0.5 & bright knot in interacting gal. \\
$0837+4717$ & HS,PC & 12630 & 18.2 & -18.1 & 7.64 & 0.77 & merger? \\
$1139+006$ & UM448 & 5560 & 14.7 & -19.9 & 7.98 & 0.5 & 2 knots in center - merger? \\
$1337-313$ & NGC5253 & 440 & 10.9 & -17.4 & 8.16 & 0.5 & Im pec, starburst \\
\hline
\end{tabular}

a data from the NED.

The scenarios of localized nitrogen enrichment, as well as the constraints imposed by the known mechanisms of fresh elements mixing and dispersal were discussed in detail by Kobulnicky et al. (1997) (see also Roy \& Kunth 1995). They concluded that, in the light of the expected short timescales for dispersal in the interstellar medium (ISM) of a few Myr, the localized nature of the $N$ enrichment suggests a very recent pollution event, probably connected with the onset of massive star winds. The alternative analysis of Tenorio-Tagle (1996) suggests the timescale of $\sim 100$ Myr to mix fresh metals from a cluster of massive stars with the gas in a disk galaxy. However, this can be barely applicable for very complex flow patterns expected in the vicinity of merger starbursts. In particular, the very rare occurrence of objects with a large $N$ overabundance, similar to that observed in Mkn 996 and HS 0837+4717, favors the general idea of the both short-time scales: for localized $N$ pollution and its fast dispersal.

It is worth noting that a selection effect, related to the starburst strength and its trigger mechanism, will affect the observed frequency of this phenomenon, probably determining the overall scale of the polluted region. In particular, the large $N$ excess in the central starbursts of NGC 5253 would be barely detected with ground-based spectroscopy, if this galaxy was a few times more distant.

\subsection{The global environment}

On the large scales, the volume around HS 0837+4717 is well sampled only by the objects from the Updated Zwicky Catalog (Falco et al. 1999), which is complete to $B=15$.5. At the BCG distance they all are luminous, with $M_{\mathrm{B}} \lesssim-20 \mathrm{~m} 6$, and are located outside the sphere with $R=8.4 \mathrm{Mpc}$, centered at HS $0837+4717$. This implies that HS $0837+4717$ is situated in a void (as several other XMD BCGs are, Pustilnik et al. 2003a). New data on the distribution of fainter galaxies with measured redshifts from the SDSS DR1 database (Abazajian et al. 2003) around HS $0837+4717$ show no objects within this sphere, corroborating the classification HS $0837+4717$ as a void galaxy.

\subsection{HS $0837+4717$ in relation to other XMD BCGs}

One of the goals of detailed studies of the properties of individual XMD BCGs is to establish how homogeneous is this group of galaxies. Current models suggest various evolution scenarios that would result in a large metal deficiency of a galaxy's ISM. They include both a significant loss of fresh metals via galactic superwinds, related to strong starbursts, and/or the infall of unprocessed intergalactic matter onto normally evolved galaxy. The third option is a very slow astration due to very low surface gas density and the stabilizing role of the Dark Matter (DM) halos (as, e.g., for LSB galaxies). Finally, one of the most intriguing options is that of truly young XMD galaxies.

Known XMD galaxies can, in principle, be the products of any of these evolutionary scenarios. Therefore, their properties could show significant diversity. HS $0837+4717$ is, in this context, an outlier among XMD BCGs and could yield important information on alternative evolutionary scenarios.

What are the main differences between HS 0837+4717 and prototypical XMD BCGs I Zw 18 and SBS 0335-052E, both known to show WR features (Sect. 4.3), but not an excess nitrogen abundance? The BCG discussed here, is the most luminous and is probably the largest galaxy in this group. The latter implies a rather massive baryon and DM configuration, capable in the isolated state of maintaining a large fraction of the newly produced metals. Its value of $M(\mathrm{HI}) / L_{\mathrm{B}} \lesssim 0.9$ implies either significant processing of neutral gas, or a very strong starburst, or both. Also, a significant gas mass fraction could be hidden in molecular form. The latter is more characteristic of luminous IR galaxies, a class of galaxies tightly connected to powerful starbursts due to the merging of gas-rich galaxies. The strong starbursts, in turn, occur when they encompass a significant part of the galaxy gas mass, what is expected in case of strong disturbances. Merging of two galaxies provides the strongest possible disturbance. This allows the breaking of the gas equilibrium even in very stable configurations, and results in the efficient gas sinking into the dynamical center of merged object with a subsequent intense SF burst (e.g., Mihos \& Hernquist 1996).

The important aspect of star formation in a merger is related to the processes taking place in the pre-merger phase. As shown by Elmegreen et al. (1993), the interstellar gas of pre-merger galaxy is highly agitated. This results in a significant increase of the Jeans mass for forming gravitationally bound molecular clouds (up to $10^{8}-10^{9} M_{\odot}$ ). The latter could be progenitors of the HS $0837+4717$ very massive starbursts. As discussed, e.g., by Elmegreen (1999), in regions with very high SF activity one should expect a shift of the IMF to higher 
masses. Similarly, due to the lower cooling efficiency at very low metallicity, the Jeans mass is expected to be higher, again resulting in an upward shift of the respective IMF. This could result in a significant increase of the relative fraction of massive stars, and a larger effect of WR stars on the local nitrogen enrichment. Thus, the option of merging of two slowly evolving XMD LSB galaxies could be a plausible explanation for the XMD BCGs with such atypical properties.

\subsection{Summary and conclusions}

We performed a complex study of one of the very metaldeficient BCG HS $0837+4717$. The long-slit high $S / N$ ratio spectrophotometry was complemented by a study of the ionized gas velocity distribution, by $\mathrm{H}$ I radio line $21-\mathrm{cm}$ total flux measurements and by broad-band imaging photometry. The observed properties of this object are extreme in several aspects.

This is the most luminous BCG with a metallicity less than or of the order of $Z_{\odot} / 20$. Its observed unusual colours are caused mainly by the very strong [O III] emission lines shifted into the $V$-band due to its significant redshift. The factor of 6 nitrogen overabundance in its supergiant H II region, in comparison to other very metal-deficient BCGs, implies either a nontypical evolution track for HS $0837+4717$, or some very short transitional phase of star formation, or both. The ionized gas kinematics appears rather disturbed. There is an indication of either counter-rotation, or of a supershell. The BCG overall disturbed optical morphology with the "double-nucleus" central structure, and two small appendages at its periphery, may support the hypothesis of an advanced merger. Recent images of this BCG from the SDSS DR1 database corroborate the described appearance.

Among the known BCGs with the well measured N/O, there are two objects with the larger $N$ excesses, and three more BCGs with an excess of a factor of 3 relative to the norm for this type of objects. All of them are WR galaxies; almost all belong to the group of luminous BCGs; and all can be interpreted as various stages of mergers. This suggests that HS $0837+4717$ could presumably be the most metal-poor representative of this group.

From the results and discussion above we draw the following conclusions:

1. The oxygen abundance in HS $0837+4717$ is $12+$ $\log (\mathrm{O} / \mathrm{H})=7.64$. The abundance ratios $\mathrm{X} / \mathrm{O}$ for the elements $\mathrm{Ne}, \mathrm{Ar}, \mathrm{S}, \mathrm{Cl}$ and $\mathrm{Fe}$ are consistent with the average values found for low-metallicity BCGs by Izotov \& Thuan (1999), implying the primary origin of these elements along with oxygen.

2. HS $0837+4717$ is the most metal-deficient galaxy among the luminous BCGs with $M_{\mathrm{B}} \leq-18$. 0 . This BCG with its $\mathrm{O} / \mathrm{H}$ and $M_{\mathrm{B}}$ significantly deviates from the $Z-L_{\mathrm{B}}$ relationship derived for the large BCG sample.

3. We detected the broad emission features characteristic of WR stars, and estimated the number of WR stars as $\sim 1000$. Their ratio to the number of O-stars is well compatible with that predicted by the current models.
4. Broad low-contrast components of the $\mathrm{H} \alpha, \mathrm{H} \beta$, and [O III] $\lambda \lambda 4959,5007$ emission lines were detected, with FWHMs of $\sim 1500 \mathrm{~km} \mathrm{~s}^{-1}$. The flux ratio of the $\mathrm{H} \alpha$ and $\mathrm{H} \beta$ broad components implies very strong obscuration ( $\sim 5 \mathrm{mag}$ in $B$-band) in the emission region or along the line of sight. The extinction-corrected luminosity of the BCG should be higher than that observed, at least, by a factor of 2.5-5.

5. The position-velocity diagrams for the $\mathrm{H} \alpha$ narrow component are not compatible with regular rotation. The major axis $\mathrm{P}-\mathrm{V}$ diagram indicates either counter-rotation, or the presence of a giant supershell with a diameter of $\sim 3.5 \mathrm{kpc}$ and an expansion velocity of about $70 \mathrm{~km} \mathrm{~s}^{-1}$. Half of the $\mathrm{P}-\mathrm{V}$ diagram can be interpreted as rotation with an amplitude of $\sim 50-70 \mathrm{~km} \mathrm{~s}^{-1}$ at a radius of $\sim 4 \mathrm{kpc}$.

6. We find that this BCG is asymmetric both near the center (the inner structure consisting of two compact regions $\sim 2 \mathrm{kpc}$ apart) and at the periphery. While the disturbed morphology of HS $0837+4717$ could suggest a recent strong interaction, no candidate galaxies are found in its vicinity. Along with the very disturbed gas kinematics this could be evidence for a hypothesis of a recent merging of two gas-rich dwarfs.

7. The nitrogen-to-oxygen abundance ratio in HS $0837+4717$ is six times higher than that of other XMD BCGs. The properties common to HS $0837+4717$ and five other nonXMD BCGs with the large nitrogen abundance excesses, suggest that the large nitrogen overabundance could be connected with merger events and with a short phase of the related powerful starburst, when many WR stars contribute to the ISM enrichment.

8. HS $0837+4717$ is located in a region with very low density of galaxies (void), the nearest of them with known redshift being situated at $\sim 6.4 \mathrm{Mpc} \mathrm{h}^{-1}$. The strong isolation of the BCG or its progenitor(s) might be the reason for its slow chemical evolution.

Acknowledgements. We are grateful to the referee D. Kunth for useful suggestions, which allowed to improve the analysis and results presentation. The authors acknowledge the partial support from INTAS grants 96-0500 and 97-0033. SAO authors acknowledge the partial support from the Russian Federal program "Astronomy". Y.I. thanks the support of National Science Foundation grant AST 02-05785 and of the Swiss SCOPE 7UKPJ62178 grant. N.B. acknowledges support from the Israel Science Foundation and from Austrian Friends of Tel Aviv University. This research has made use of the NASA/IPAC Extragalactic Database (NED), which is operated by the Jet Propulsion Laboratory, California Institute of Technology, under contract with the National Aeronautics and Space Administration. The SDSS DR1 database is completed for public use thanks to the funding by the Alfred P. Sloan Foundation, the SDSS member institutions, the National Aeronautics and Space Administration, the National Science Foundation, the U.S. Department of Energy, the Japanese Monbukagakusho, and the Max Planck Society. The SDSS Web site is http://www. sdss.org/.

\section{References}

Abazajian, K., Adelman-McCarthy, J. K., Agueros, M. A., et al. 2003, AJ, 126, 2081 
Afanasiev, V. L., Burenkov, A. N., Vlasyuk, V. V., \& Drabek, S. V. 1995, SAO RAS internal report, No. 234

Aller, L. H. 1984, Physics of Thermal Gaseous Nebulae (Dordrecht: Reidel)

Amram, P., \& Östlin, G. 2001, Messenger, 103, 31

Bergvall, N., \& Östlin, G. 2002, A\&A, 390, 891

Bohlin, R. C. 1996, AJ, 111, 1743

Chamberlain, J. W. 1953, ApJ, 117, 387

Elmegreen, B. G. 1999, in The evolution of Galaxies on Cosmological Timescales, ed. J. E. Beckman, \& T. J. Mahoney, ASP Conf. Ser., 187,145

Elmegreen, B. G., Kaufman, M., \& Thomasson, M. 1993, ApJ, 412, 90

Falco, E., Kurtz, M., Geller, M., et al. 1999, PASP, 111, 438

Fioc, M., \& Rocca-Volmerange, B. 1997, A\&A, 326, 950

Fricke, K., Izotov, Y. I., Papaderos, P., Guseva, N. G., \& Thuan, T. X. 2001, AJ, 121, 169

Garnett, D. R. 1992, AJ, 103, 1330

Genzel, R., Tacconi, L. J., Rigopoulou, D., Lutz, D., \& Tecza, M. 2001, ApJ, 563, 527

Guseva, N. G., Izotov, Y. I., \& Thuan, T. X. 2000, ApJ, 531, 776

Hunt, L., Vanzi, L., \& Thuan, T. X. 2001, A\&A, 377, 66

Hunter, D. A., \& Gallagher, J. S. 1986, PASP, 98, 5

Izotov, Y. I., \& Thuan, T. X. 1999, ApJ, 511, 639

Izotov, Y., Thuan, T. X., \& Lipovetsky, V. 1994, ApJ, 435, 647

Izotov, Y. I., Dyak, A. B., Chaffee, F., et al. 1996, ApJ, 458, 524

Izotov, Y., Thuan, T. X., \& Lipovetsky, V. 1997a, ApJS, 108, 1

Izotov, Y. I., Lipovetsky, V. A., Chaffee, F., et al. 1997b, ApJ, 476, 298

Izotov, Y. I., Foltz, C. B., Green, R. F., Guseva, N. G., \& Thuan, T. X. 1997c, ApJ, 487, L37

Izotov, Y. I., Chaffee, F. H., Foltz, C., et al. 1999, ApJ, 527, 757

Izotov, Y. I., Chaffee, F. H., \& Schaerer, D. 2001, A\&A, 378, L45

Kniazev, A. Y., Pustilnik, S. A., Ugryumov, A. V., \& Kniazeva, T. F. 2000a, Astron. Lett., 26, 129

Kniazev, A., Pustilnik, S., Masegosa, J., et al. 2000b, A\&A, 357, 101

Kniazev, A. Y., Pustilnik, S. A., Pramsky, A. G., Ugryumov, A. V., \& Kniazeva, T. F. 2001, A\&A, 371, 404

Kniazev, A. Y., Grebel, E. K., Hao, L., et al. 2003, ApJ, 593, L73

Kobulnicky, H. A., \& Skillman, E. D. 1996, ApJ, 471, 211

Kobulnicky, H. A., \& Skillman, E. D. 1997, ApJ, 489, 636

Kobulnicky, H. A., Skillman, E. D., Roy, J.-R., Walsh, J. R., \& Rosa, M. R. 1997, ApJ, 477, 679

Kunth, D., \& Joubert, M. 1985, A\&A, 142, 411

Kunth, D., \& Östlin, G. 2000, A\&AR, 10, 1

Landolt, A. 1992, AJ, 104, 340
Legrand, F., Kunth, D., Roy, J.-R., Mas-Hesse, J. M., \& Walsh, J. R. 1997, A\&A, 326, L17

Leitherer, C. 1990, ApJS, 73, 1

Leitherer, C., Schaerer, D., Goldader, J. D., et al. 1999, ApJS, 123, 3

Massey, P., Strobel, K., Barnes, J. V., \& Anderson, E. 1988, ApJ, 328, 315

Mihos, J. C., \& Hernquist, L. 1996, ApJ, 464, 641

Östlin, G., Amram, P., Bergvall, N., Masegosa, J., \& Boulesteix, J. 1999, A\&AS, 147, 419

Östlin, G., Amram, P., Bergvall, N., et al. 2001, A\&A, 374, 800

Pagel, B., Terlevich, R., \& Melnick, J. 1986, PASP, 98, 1005

Pagel, B. E. J., Simonson, E. A., Terlevich, R. J., \& Edmunds, M. G. 1992, MNRAS, 255, 325

Pustilnik, S. A., Engels, D., Ugryumov, A. V., et al. 1999, A\&AS, 137, 299

Pustilnik, S. A., Brinks, E., Thuan, T. X., Lipovetsky, V. A., \& Izotov, Y. I. 2001a, AJ, 121, 1413

Pustilnik, S. A., Kniazev, A. Y., Lipovetsky, V. A., \& Ugryumov, A. V. 2001b, A\&A, 373, 24

Pustilnik, S. A., Kniazev, A. Y., Pramskij, A. G., Ugryumov, A. V., \& Masegosa, J. 2003a, A\&A, 409, 917

Pustilnik, S., Kniazev, A., Ugryumov, A., \& Pramskij, A. 2003b, Ap\&SS, 284, 795

Roy, J.-R., \& Kunth, D. 1995, A\&A, 294, 432

Salzer, J. J., Di Serego Alighieri, S., Matteucci, F., Giovanelli, R., \& Haynes, M. 1991, AJ, 101, 1258

Schaerer, D., \& Vacca, W. D. W. 1998, ApJ, 497, 618

Schaerer, D., Contini, T., \& Kunth, D. 1999, A\&A, 341, 399

Stasińska, G. 1990, A\&AS, 83, 501

Telles, E., \& Terlevich, R. 1997, MNRAS, 286, 183

Tenorio-Tagle, G. 1996, AJ, 111, 1641

Tenorio-Tagle, G., \& Bodenheimer, P. 1988, ARA\&A, 26, 145

Thuan, T. X., \& Izotov, Y. I. 1997, ApJ, 489, 623

Thuan, T. X., Izotov, Y., \& Lipovetsky, V. 1996, ApJ, 463, 120

Ugryumov, A. V., Engels, D., Pustilnik, S. A., et al. 2003, A\&A, 397, 463

Vacca, W. D. 1994, ApJ, 421, 140

Vacca, W. D., \& Conti, P. S. 1992, ApJ, 401, 543

Weaver, R., McCray, R., Castor, J., Shapiro, P., \& Moore, R. 1977, ApJ, 218, 377

Whitford, A. E. 1958, AJ, 63, 201

York, D., Adelman, J., Anderson, J., et al. 2000, AJ, 120, 1579

Zasov, A., Kniazev, A., Pustilnik, S., et al. 2000, A\&AS, 144, 429

van Zee, L., Westpfahl, D., Haynes, M., \& Salzer, J. J. 1998, AJ, 115, 1000 